\title{
Polysaccharide biosynthetic pathway profiling and putative gene mining of Dendrobium moniliforme using RNA-Seq in different tissues
}

\author{
Yingdan Yuan ${ }^{1,2}$, Jinchi Zhang ${ }^{1,2^{*}}$ (D) Justin Kallman ${ }^{3}$, Xin Liu ${ }^{1,2}$, Miaojing Meng ${ }^{1,2}$ and Jie Lin ${ }^{1,2}$
}

\begin{abstract}
Background: Dendrobium moniliforme (Linnaeus) Swartz is a well-known plant used in traditional Chinese medicine due to bioactive constituents. Polysaccharides are the main medicinal ingredients, yet no studies have been published on polysaccharide biosynthesis in $\mathrm{D}$. moniliforme. To comprehensively investigate the polysaccharide at the transcription level, we performed de novo transcriptome sequencing for the first time to produce a comprehensive transcriptome of D. moniliforme.
\end{abstract}

Results: In our study, a database of 562,580 unigenes (average length $=1115.67$ bases) was generated by performing transcriptome sequencing. Based on the gene annotation of the transcriptome, we identified 1204 carbohydrate-active related unigenes against CAZy database, including 417 glycosyltransferase genes (GTs), 780 glycoside hydrolases (GHs), 19 carbohydrate esterases (CEs), 75 carbohydrate-binding modules (CBMs), and 44 polysaccharide lyases (PLs). In the cellulose synthase family, 21 differential expression genes (DEGs) related to polysaccharide were identified. Subsequently, the tissue-specific expression patterns of the genes involved in polysaccharide pathway were investigated, which provide understanding of the biosynthesis and regulation of DMP at the molecular level. The two key enzyme genes (Susy and SPS) involved in the polysaccharide pathway were identified, and their expression patterns in different tissues were further analyzed using quantitative real-time PCR

Conclusions: We determined the content of polysaccharides from Dendrobium moniliforme under different tissues, and we obtained a large number of differential genes by transcriptome sequencing. This database provides a pool of candidate genes involved in biosynthesis of polysaccharides in D. moniliforme. Furthermore, the comprehensive analysis and characterization of the significant pathways are expected to give a better insight regarding the diversity of chemical composition, synthetic characteristics, and the regulatory mechanism which operate in this medical herb.

Keywords: Dendrobium moniliforme, Transcriptome, Polysaccharides synthesis, Glycosyltransferase

\section{Background}

Dendrobium moniliforme (Linnaeus) Swartz, is one of the most widespread species in the Orchidaceae family, which is a widely cultivated medicinal crop around Asia [1]. D. moniliforme has the characteristics of a thin stem, small flower, and it is a representative plant of Chinese traditional

\footnotetext{
*Correspondence: zhangjc8811@gmail.com

${ }^{1}$ Co-Innovation Center for Sustainable Forestry in Southern China, Nanjing Forestry University, Nanjing 210037, China

${ }^{2}$ Jiangsu Province Key Laboratory of Soil and Water Conservation and Ecological Restoration, Nanjing Forestry University, Nanjing 210037, China Full list of author information is available at the end of the article
}

medicine that is often processed into finished medicinal materials. It is widely used as folk medicine for antipyretic purpose, benefits to eyes, and as a tonic [2]. D. moniliforme plants' main active ingredients are polysaccharides, alkaloids, bibenzyls, fluorenone compounds, glycosides, amino acids and several trace mineral elements [3]. The stems of D. moniliforme are the main medicinal parts, which contain thick water-soluble polysaccharides. Polysaccharide hydrolyzate contains not only mannose and glucose, but also trace amounts of arabinose and xylose [4]. Polysaccharides have been successfully extracted from 100 kinds of plants 
and are commonly used in the research and development in medicine and health food. They have pharmacological effects of regulating immunity, and acting against tumors and oxidants. Polysaccharides of different origins exhibit different biological activities [5-7].

Polysaccharides are natural macromolecules consisting of multiple monosaccharides units. They represent a structurally diverse class of macromolecules that are widely distributed in nature and play an important role in controlling cell division, regulating cell growth and maintaining normal metabolism of living organisms. Polysaccharides of higher plants are a potential source of pharmacologically active compounds. Numerous studies have shown that polysaccharides isolated from medicinal plants could affect the immune responses both in vivo and in vitro and have the potential of being immunomodulators [8]. The polysaccharide of Dendrobium in most of the literature refers to the water-soluble polysaccharide $[9,10]$. At present, many people have studied its structure. For the currently separated polysaccharide components of Dendrobium, the monosaccharide composition consists mainly of glucose and mannose, and also contains arabinose, galacturonic acid, xylose, rhamnose, galactose and so on [11-13]. All in all, mannose and glucose are the main monosaccharides in these Dendrobium species.

The purpose of our research on medicinal ingredients is to be able to apply the extracts of these medicinal ingredients to clinical practice. In order to meet the growing demand for drugs, researchers have gradually applied genetic engineering methods to produce these medicinal components. Therefore, the biosynthesis and metabolism pathways of various active ingredients and the excavation of related key enzyme genes have become important goals of the research on medicinal plants. Functional genomics methods, especially transcriptomics, have shown important applications in discovering the key enzyme genes involved in biosynthesis of secondary metabolites in medicinal plants and elucidating secondary metabolic pathways and regulatory mechanisms. The transcriptome is based on the predecessor (mRNA) of protein in live cells, which represents the real-time situation of life activity and is a very effective method and means to reveal the molecular mechanism of biological growth and physiological activities $[14,15]$. In medicinal plant research, transcriptome sequencing technology is widely used in the discovery of new genes [16], metabolic pathways [17], molecular marker mining [18], transcription mapping [19] and so on. A large number of medicinal plants have been studied by RNA-seq transcriptomics, such as Lycium chinense [20], Salvia miltiorrhiza [21], Catharanthus roseus [22], and American ginseng [23]. However, for the first time, we sequenced the transcriptome of three tissues to reveal the polysaccharide synthesis genes and pathways in D. moniliforme.
Until now, several transcriptomes in D. officinale [24$26]$ and a few whole genomes have been sequenced [27, 28]. In Dendrobium species, a lot of key enzymeencoding genes involved in the synthesis and metabolic pathways have been identified. However, Dendrobium molecular biology research information is still very limited. The molecular marker development and utilization of key genes and other aspects of this study are still in their infancy. The molecular mechanisms underlying polysaccharide biosynthesis and the related metabolic pathways for D. moniliforme remain unknown. In this study, we constructed nine transcriptome libraries for root, stem and leaf material in D. moniliforme. A total of 1335 glycosyltransferase genes (GTs) and 35 cellulose synthase genes (CesA) were identified, and we also analyzed differentially expressed genes (DEGs) between them. Through the analysis of sequencing data, we understand the biosynthesis of secondary products, particularly in identifying candidate genes involved in DMP biosynthesis. Meanwhile, we verified the quality of our dataset and confirmed these putative DMP genes by quantitative real-time RT-PCR (qRT-PCR).

\section{Results \\ Determination of polysaccharides in three different tissues}

Polysaccharide was determined in three different tissues, including leaf, stem and root material. The stem of D. moniliforme is its main medicinal part and polysaccharide was mainly concentrated in stems. The highest content of polysaccharide was $24.53 \%$ for stems (Fig. 1). From one-way analysis of variance, it is shown that the polysaccharide content of the stem of $D$. moniliforme was significantly different from that of roots and leaves.

\section{Illumina sequencing and de novo assembly}

In this study, nine cDNA libraries were constructed from the three tissues of $D$. moniliforme in three replicates. These libraries were named as follows: root cDNA libraries: Dm_R1, Dm_R2, Dm_R3; stem cDNA libraries: Dm_ S1, Dm_S2, Dm_S3; leaf cDNA libraries: Dm_L1, Dm_L2, Dm_L3. In total, 811,451,164 clean reads with a total of $119.89 \mathrm{~Gb}$ (Gigabase) were obtained from all of the samples. The base average error rate was $0.01 \%$ and the average GC content was $46.36 \%$. The average values of Q20 and Q30 were 98.14 and $94.57 \%$, respectively (Table 1).

We combined all clean reads to form the $D$. moniliforme transcriptome database by using Trinity software. 679,321 transcripts were identified with the average transcript being 703.77 base pairs (bp). The length distribution of transcripts and unigenes is shown in Additional file 1: Figure S1. Sequencing length of transcripts and unigenes ranged from $201 \mathrm{bp}$ to $16,515 \mathrm{bp}$. Assembly of clean reads resulted in 562,480 unigenes with a 


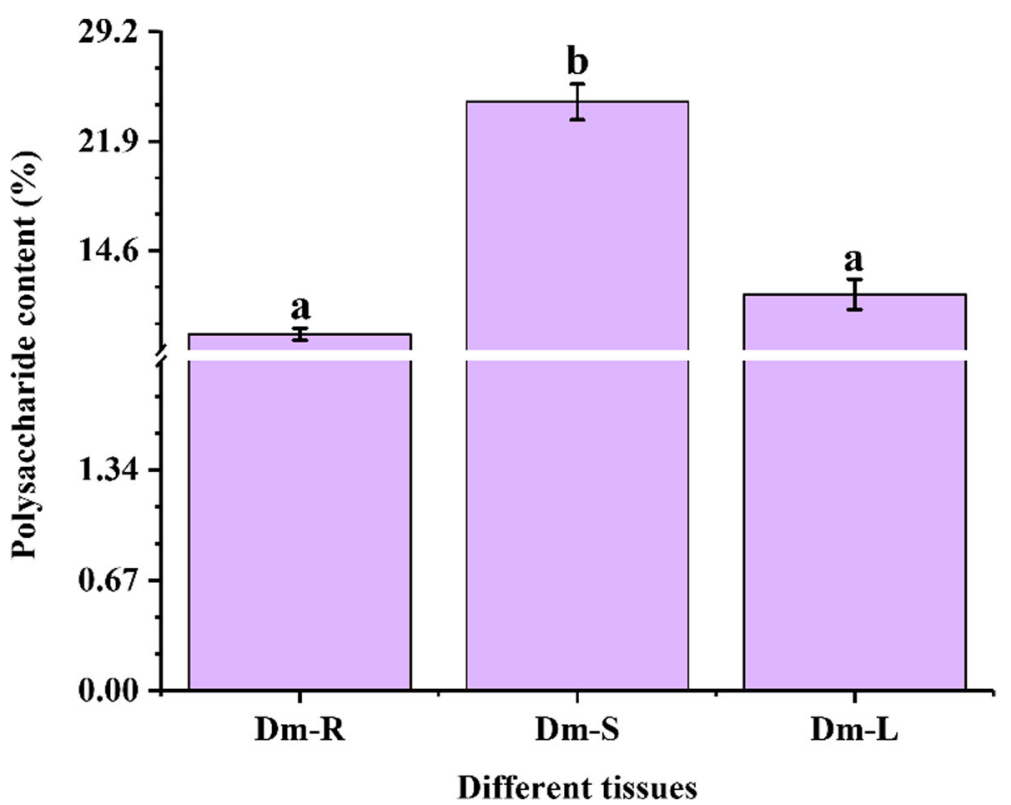

Fig. 1 Determination of polysaccharide contents in different tissues of $D$. moniliforme. The a and b letters indicate statistical differences in the results of analysis of variance between different tissues, with a significant difference of $p<0.05$

mean length of $567.79 \mathrm{bp}$. The N50 length of transcripts and unigenes were $1177 \mathrm{bp}$ and $830 \mathrm{bp}$, respectively.

\section{Functional annotation, GO, KOG and KEGG classification}

A total of 562,480 unigenes were annotated against the Nr, KEGG, Swiss-Prot, Pfam and String databases using the BLAST algorithm (E-value<1E-5) with 14,709 (4.25\%) in all five databases (Additional file 1: Figure S2). Analyses showed that 169,702 unigenes (48.99\%) had significant matches in the $\mathrm{Nr}$ database, 110,415 unigenes $(31.87 \%)$ in the Swiss-Prot database and 104,684 unigenes $(30.22 \%)$ in the KEGG database. The lowest proportion occurred in the String database $(37,245,10.75 \%)$ and the Pfam was 65,595 (18.94\%) annotated unigenes.
In the KEGG database, a total of 104,684 unigenes were annotated to 33 pathways (Fig. 2a). All unigenes were divided into five categories: Metabolism (A), Genetic Information Processing (B), Environmental Information Processing (C), Cellular Processes (D), Organismal Systems (E). The pathways involving the highest number of unigenes were "global and overview maps" (25,093), followed by "translation" $(15,778)$ and "signal transduction" $(10,348)$.

In addition, the annotated sequences were subjected to the clusters of orthologous groups (KOG) database for functional prediction and classification. There were 37, 245 unigenes assigned to KOG classification and divided into 25 specific categories involved in cellular process, signal transduction, metabolism and other processes

Table 1 Summary of sequencing quality

\begin{tabular}{|c|c|c|c|c|c|c|c|}
\hline Sample ID & Raw reads & Clean reads & Clean bases & Error (\%) & Q20 (\%) & Q30 (\%) & GC (\%) \\
\hline Dm_R1 & $104,974,434$ & $103,116,856$ & $15.24 \mathrm{~Gb}$ & 0.0126 & 98.12 & 94.61 & 46.41 \\
\hline Dm_R2 & $112,417,410$ & $110,298,744$ & $16.38 \mathrm{~Gb}$ & 0.012 & 98.43 & 95.25 & 46.63 \\
\hline Dm_R3 & $93,916,312$ & $91,971,512$ & $13.59 \mathrm{~Gb}$ & 0.0125 & 98.22 & 94.7 & 46.35 \\
\hline Dm_S1 & $95,188,100$ & $93,364,948$ & $13.79 \mathrm{~Gb}$ & 0.0129 & 97.99 & 94.31 & 46.49 \\
\hline Dm_S2 & $83,861,418$ & $81,881,046$ & $12.08 \mathrm{~Gb}$ & 0.0127 & 98.12 & 94.44 & 46.1 \\
\hline Dm_S3 & $95,368,208$ & $92,929,138$ & $13.69 \mathrm{~Gb}$ & 0.0128 & 98.05 & 94.27 & 46.07 \\
\hline Dm_L1 & $84,551,998$ & $83,097,662$ & $12.29 \mathrm{~Gb}$ & 0.0126 & 98.13 & 94.64 & 46.66 \\
\hline Dm_L2 & $77,755,976$ & $75,885,672$ & $11.18 \mathrm{~Gb}$ & 0.0127 & 98.11 & 94.43 & 46.36 \\
\hline Dm_L3 & $80,621,980$ & $78,905,586$ & $11.64 \mathrm{~Gb}$ & 0.0127 & 98.12 & 94.45 & 46.15 \\
\hline Total & $828,655,836$ & $811,451,164$ & $119.89 \mathrm{~Gb}$ & & & & \\
\hline
\end{tabular}

(1) Q20: percentage of bases with a Phred value > 20; Q30: percentage of bases with a Phred value > 30. (2) Error (\%): Base error rate. (3) GC (\%): percentage of bases $\mathrm{G}$ and $\mathrm{C}$ number in the total number of bases 


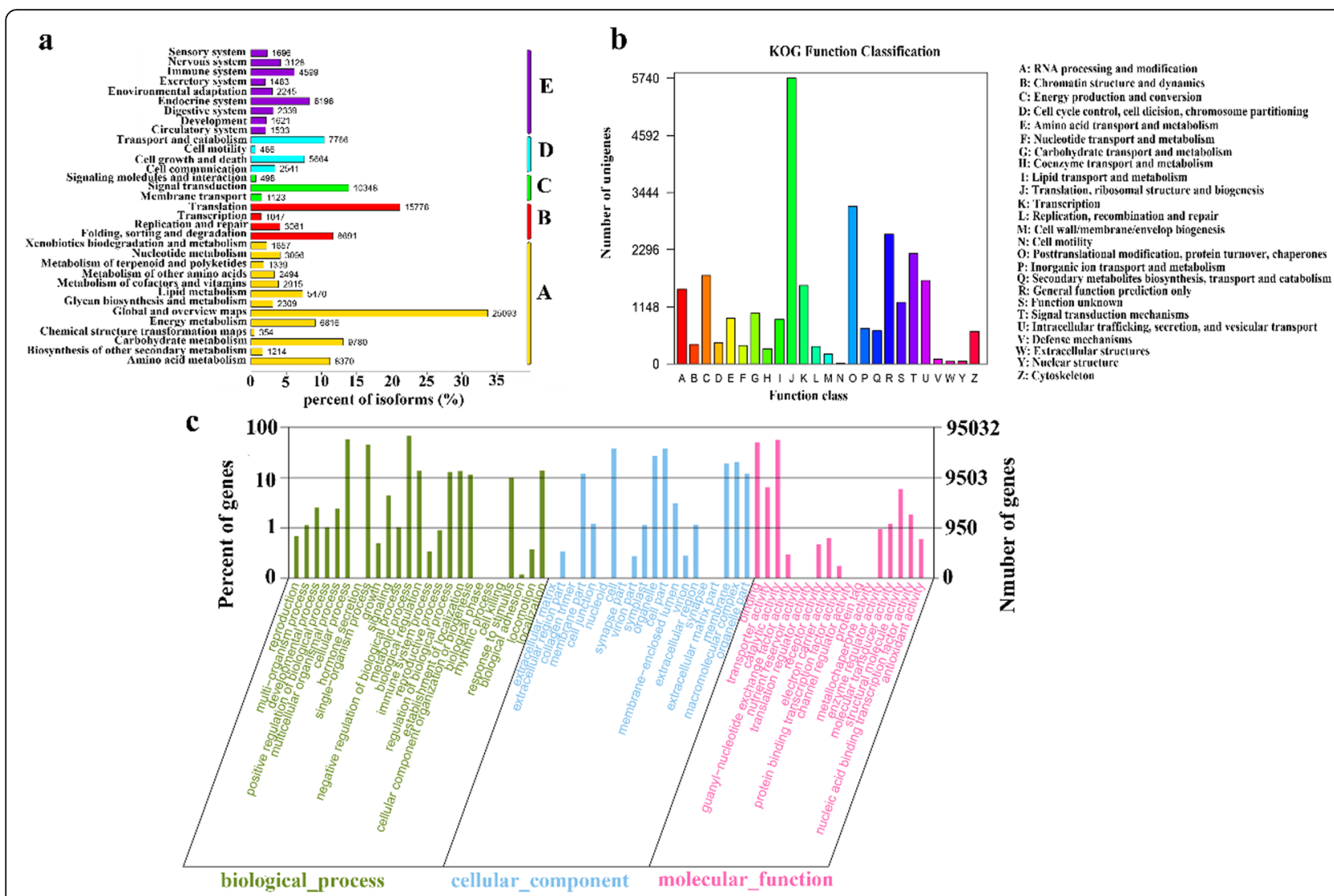

Fig. 2 Classification of unigenes and metabolic pathways. a KEGG classification of metabolic pathways. The x-axis indicates the percentage of the number of genes annotated to the pathway out of the total number of genes annotated. The $y$-axis indicates the name of the KEGG metabolic pathway. The genes were divided into five branches according to the KEGG metabolic pathway: Metabolism (A), Genetic Information Processing (B), Environmental Information Processing (C), Cellular Processes (D), Organismal Systems (E). b KOG classification of unigenes. The x-axis indicates the name of the 25 groups of KOG. The $y$-axis indicates the percentage of the number of genes annotated to the group out of the total number of genes annotated. $\mathbf{c ~ K O G}$ classification of unigenes. The $\mathrm{x}$-axis indicates the name of the 25 groups of KOG. The $y$-axis indicates the percentage of the number of genes annotated to the group out of the total number of genes annotated

(Fig. 2b). The three top terms were (J) translation, ribosomal structure and biogenesis, $(\mathrm{O})$ posttranslational modification, protein turnover, chaperones and $(\mathrm{R})$ general function prediction only.

For GO analysis, a total of 95,032 annotated unigenes were classified into three main ontologies and 62 subcategories by using Blast2Go (Fig. 2c). For the biological process (BP) category, genes involved in "metabolic process" $(63,996)$ were high represented. Furthermore, cellular component $(\mathrm{CC})$ categories mainly comprised proteins involved in "cell" $(36,019)$ and "cell part" $(36,018)$. In addition, highly represented in molecular function (MF) categories were "catalytic activity" (52,948) and "binding" $(47,656)$.

\section{Identification and cluster analysis of differentially expressed genes (DEGs)}

In this study, we examined differential expression of replicated count data by using edgeR. We set "FDR $<0.05 \&$ $\left|\log _{2} \mathrm{FC}\right| \geq 1$ " as the standard for significant differences in the expression of genes. When the $\log _{2} \mathrm{FC}>1$, the
DEG was considered as up-regulated. On the contrary, for $\log _{2} \mathrm{FC}<-1$, it was considered as down-regulated. To study gene expression among different tissues, we identified 9881 DEGs between Dm_L and Dm_S, including 7788 up-regulated and 2093 down-regulated DEGs. Comparing Dm_R with Dm_L, the largest number of DEGs were identified. Of these DEGs, 277,625 were down-regulated and 3987 were up-regulated. Finally, there were 15,834 DEGs between Dm_R and Dm_S, 2389 of which were up-regulated and 13,445 of which were down-regulated (Fig. 3d). Furthermore, 2294 and 395 DEGs were expressed uniquely in "Dm_L vs. Dm_S" and "Dm_R vs. Dm_S", respectively. Also, 11,193 DEGs were expressed uniquely between $\mathrm{Dm} \_\mathrm{R}$ and $\mathrm{Dm} \_\mathrm{L}$. Among these libraries, 891 DEGs were identified in common (Fig. 3a-c). All the details of DEGs are shown in the Additional files 2, 3 and 4.

Based on the transcriptome data, the hierarchical clustering of DEGs (Fig. 4a) between different tissues indicated the abundance of DEGs by combining the FPKM 

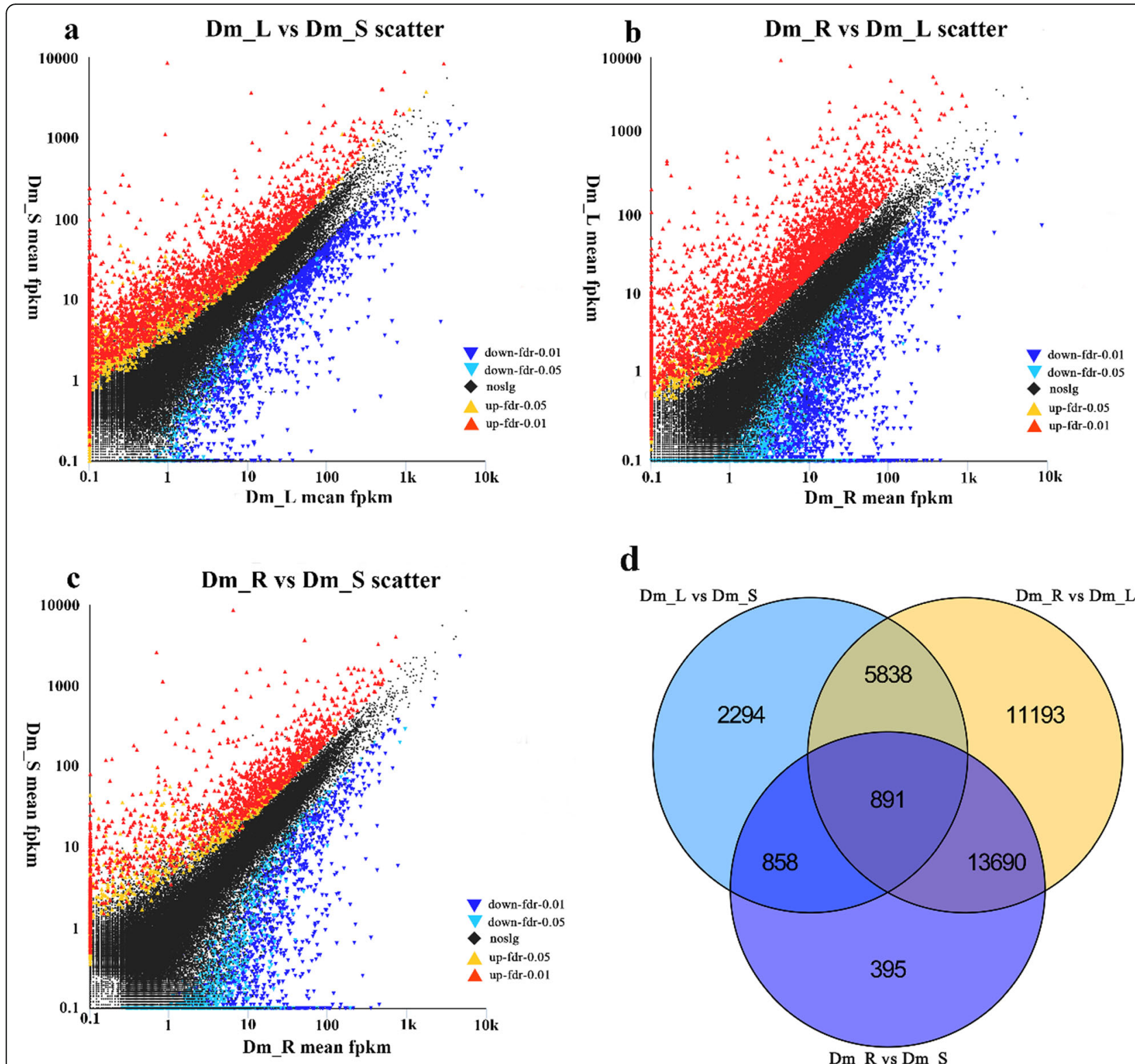

d

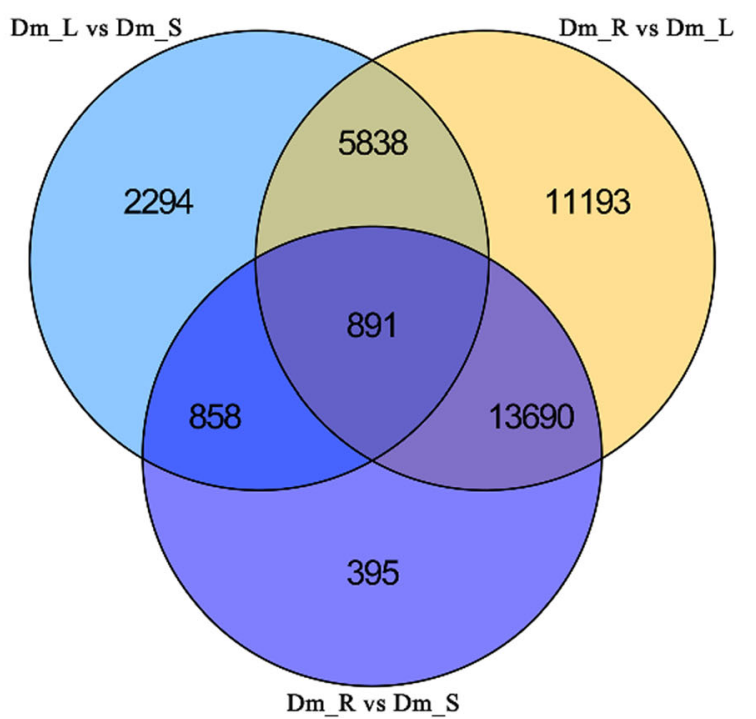

Fig. 3 a-c Scatter plots of the DEGs in different comparisons. The red dots mean significantly up-regulated genes and the blue dots represent significantly down-regulated genes. The black dots represent non-DEGs. a Dm_L vs. Dm_S scatter; b Dm_R vs. Dm_L scatter; c Dm_R vs. Dm_S scatter. $\mathbf{d}$ Venn diagram of differentially expressed genes (DEGs) in different comparisons. All DEGs are clustered into three comparison groups represented by three ellipses. The overlapping parts of different ellipses represent the number of DEGs in common from those comparison groups

with color. A total of 35,159 DEGs were identified and analyzed using criteria of $\log 10(\mathrm{FPKM}+1)$ and $p<0.05$. For the trend of the specific expression level, see the numbers under the color bar at the top left. The left is the tree of the gene cluster; the closer the two genes are separated, the closer they are expressed.

To reflect the major trends and tissue-specific expression between tissues in D. moniliforme, all DEGs were clustered into ten expression profiles (Fig. 4b) by the K-means method and hierarchical clustering with similar regulation model and $\log 2$ (foldchange). Unigenes belonging to cluster
4, 6 and 8 were more highly expressed in stems than in other tissues; unigenes belonging to cluster 3 and 5 were highly expressed in leaves. Interestingly, all unigenes mainly expressed in roots belong to a cluster of 2256 genes.

\section{Enrichment analysis and metabolic pathway assignment by KEGG}

Further, to gain a better understanding of the function clusters and biochemical pathways, we performed GO and KEGG enrichment analysis on all DEGs. In detail, 2188 GO terms were identified in "Dm_L vs. Dm_S" comparisons; 
a
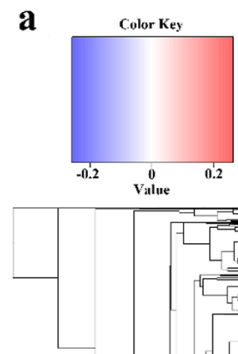

$+2$

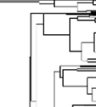

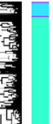
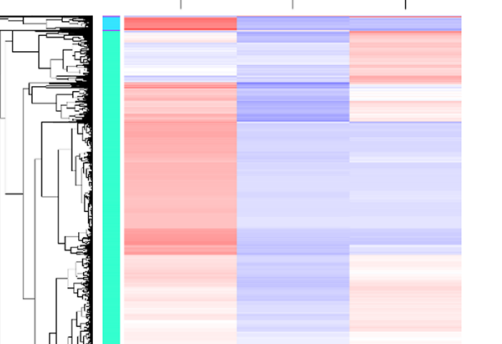

b
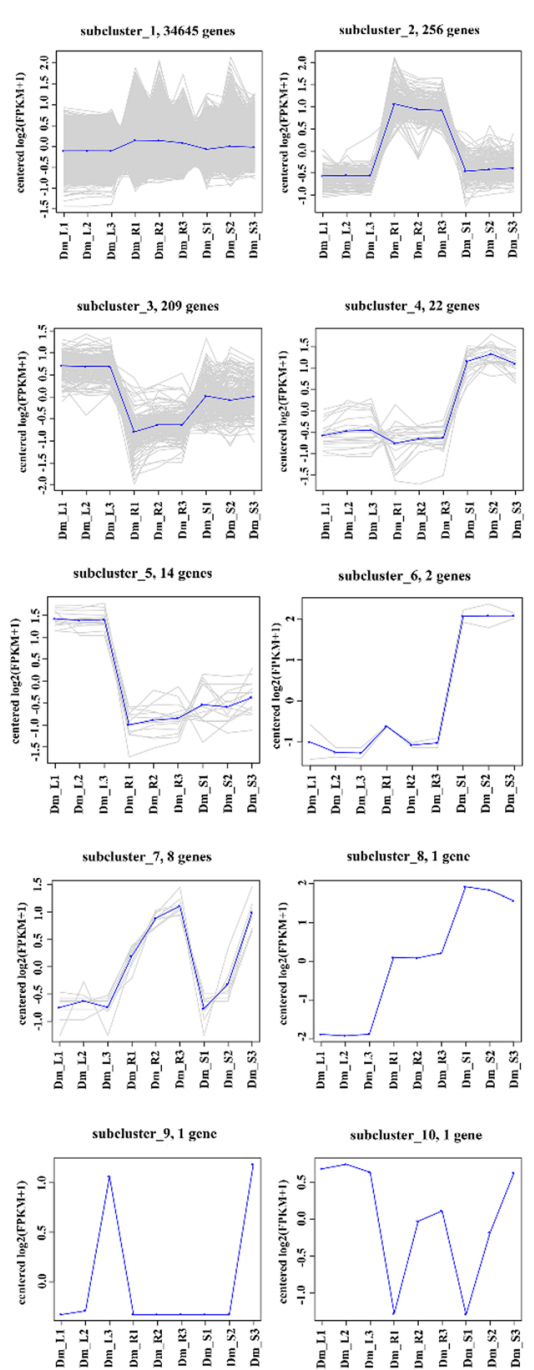

Fig. 4 Expression profiles of all DEGs. a Heat map of DEGs by hierarchical cluster analysis and FPKM distribution of all unigenes. Each column in the figure shows one sample, each row represents one gene. The color in the figure indicates the size of the gene expression in the sample $(\log 10($ FPKM +1$))$. Red indicates that the gene is highly expressed in the sample, and the blue indicates a lower expression level. The top of the tree is a cluster of samples, with the name of the sample below. $\mathbf{b}$ Cluster analysis by K-means method from gene expression profiles. The blue lines show expression model. The gray lines are all individual DEGs' expression profiles. The x-axis represents the different tissues of Dendrobium moniliforme. The $y$-axis represents $\log _{2}$ (ratio)

2691 GO terms were identified in "Dm_R vs. Dm_L" comparisons; while 1820 GO terms were identified in " $\mathrm{Dm} \_\mathrm{R}$ vs. Dm_S" comparisons. A figure for GO significant enrichment is shown in Additional file 1: Figure S3. By visualizing the GO term from GO enrichment analysis, we can see the correlation between these functions. The structure of GO can be described in the form of a directed acyclic graph (DAG) in which each GO term is a node and the parentage is an arrow. A thumbnail view of the directed acyclic graph (DAG) on molecular function between $\mathrm{Dm}_{-} \mathrm{R}$ vs. $\mathrm{Dm} \_\mathrm{S}$ is shown in Fig. 5. The top three GO terms, shown in expanded size, are polysaccharide biosynthesis process (GO: 0000271), cellular polysaccharide biosynthesis process (GO:
0033692) and cellular carbohydrate biosynthesis process (GO:0034637). Polysaccharides are the main medicinal composition of D. moniliforme, the result of GO enrichment is the same to it.

In the KEGG enrichment analysis (Additional file 1: Figure S4), KEGG pathway was divided into seven classes: Environmental Information Processing (EIP), Genetic Information Processing (GIP), Cellular Processes (CP), Organismal Systems (OS), Drug Development (DD), Human Diseases (HD) and Metabolism (M). In terms of the KEGG pathways, "Dm_L vs. Dm_S" comparisons are involved in 332 pathways with 8279 DEGs and 249,623 background unigenes. In "Dm_R vs. Dm_L" 


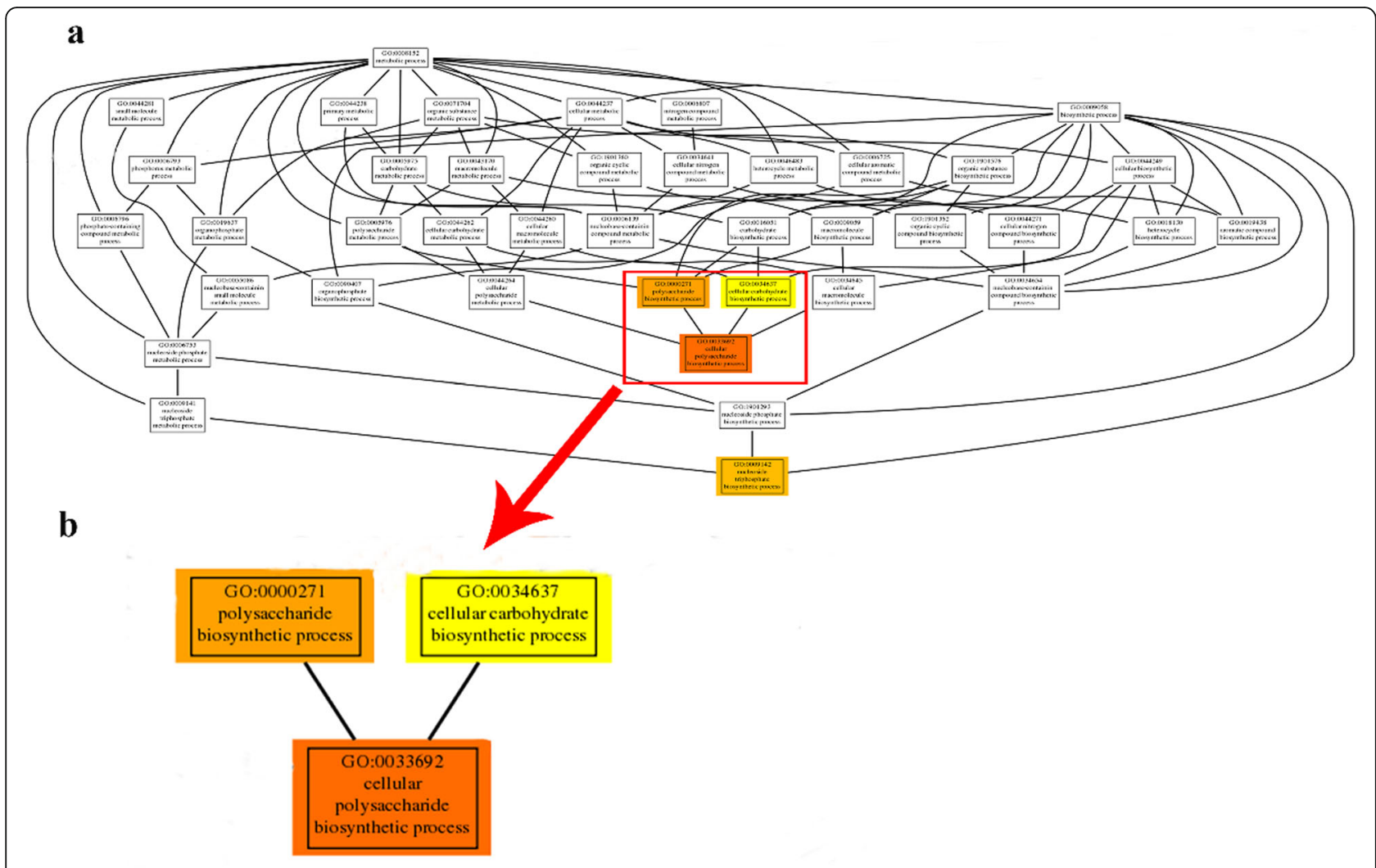

Fig. 5 Thumbnails view of directed acyclic graph (DAG) on Molecular function between Dm_R vs. Dm_S. a Thumbnails view of DAGs on MF. The depth of the color represents the degree of enrichment, the deeper the color represents the higher the degree of enrichment. The node horizontal position means the depth of GO terms. $\mathbf{b}$ Information of three top enriched terms

comparisons, 351 pathways were involved in and 32,085 DEGs and 250,769 background unigenes were identified. Finally, in "Dm_R vs. Dm_S" comparisons, 343 pathways were involved, and 17,090 DEGs and 250,453 background unigenes were identified. In general, when the corrected $p$-value $<0.05$, the GO and KEGG enrichment is considered to be significant. The top 20 KEGG enrichment pathways are shown in Fig. 6.

Plant secondary metabolites play an important role in many aspects of plant life activities, and many plant secondary metabolites are necessary for plant life activities [29]. In this study, a total of 104,684 unigenes were assigned to 503 KEGG pathways. Among these pathways, 20 KEGG pathways related to secondary metabolites were identified, including anthocyanin biosynthesis (ko00942), betalain biosynthesis (ko00965), brassinosteroid biosynthesis (ko00 905), flavonoid biosynthesis (ko00944), caffeine metabolism (ko00232), monobactam biosynthesis (ko00261) and others (Table 2). The phenylpropanoid biosynthesis (698 unigenes) pathway represented the largest unigenes group, followed by steroid biosynthesis (626 unigenes) and cyanoamino acid metabolism (600 unigenes). These pathways may provide resources for future study of the functions and processes during $D$. moniliforme development.

\section{Detection of candidate genes related to} glycosyltransferases and cellulose synthase in $D$. moniliforme

The carbohydrate-active enzyme database (CAZy) is a database resource for enzymes that can synthesize or disassemble complex carbohydrates and glycoconjugates. The carbohydrate active enzymes by functional classification include glycosyltransferase genes (GTs), glycoside hydrolases (GHs), carbohydrate esterases (CEs), carbohydrate-binding modules (CBMs) and polysaccharide lyases (PLs). We identified 1204 carbohydrate-active related unigenes against CAZy database using BLASTX $(\mathrm{E}<0.00001)$, which include 417 GTs, 780 GHs, 19 CEs, 75 CBMs and 44 PLs (Fig. 7).

Glycosyltransferase is found in almost all organisms and catalyze the transfer of glycosyl groups, which is one of the most important for biotransformation and is directly involved in the biosynthesis of disaccharides, monosaccharides, oligosaccharides, alkyl glucosides and polysaccharides [30]. Among 417 glycosyltransferase genes (GTs), we identified several DEGs of GTs that exist in these three comparisons (Table 3). GTs account for a large proportion of the carbohydrate-related family. In the Dm_R vs. Dm_L comparison, 57 DEGs were identified including 7 up-regulated and 50 down-regulated of GTs. In fucosyltransferases 


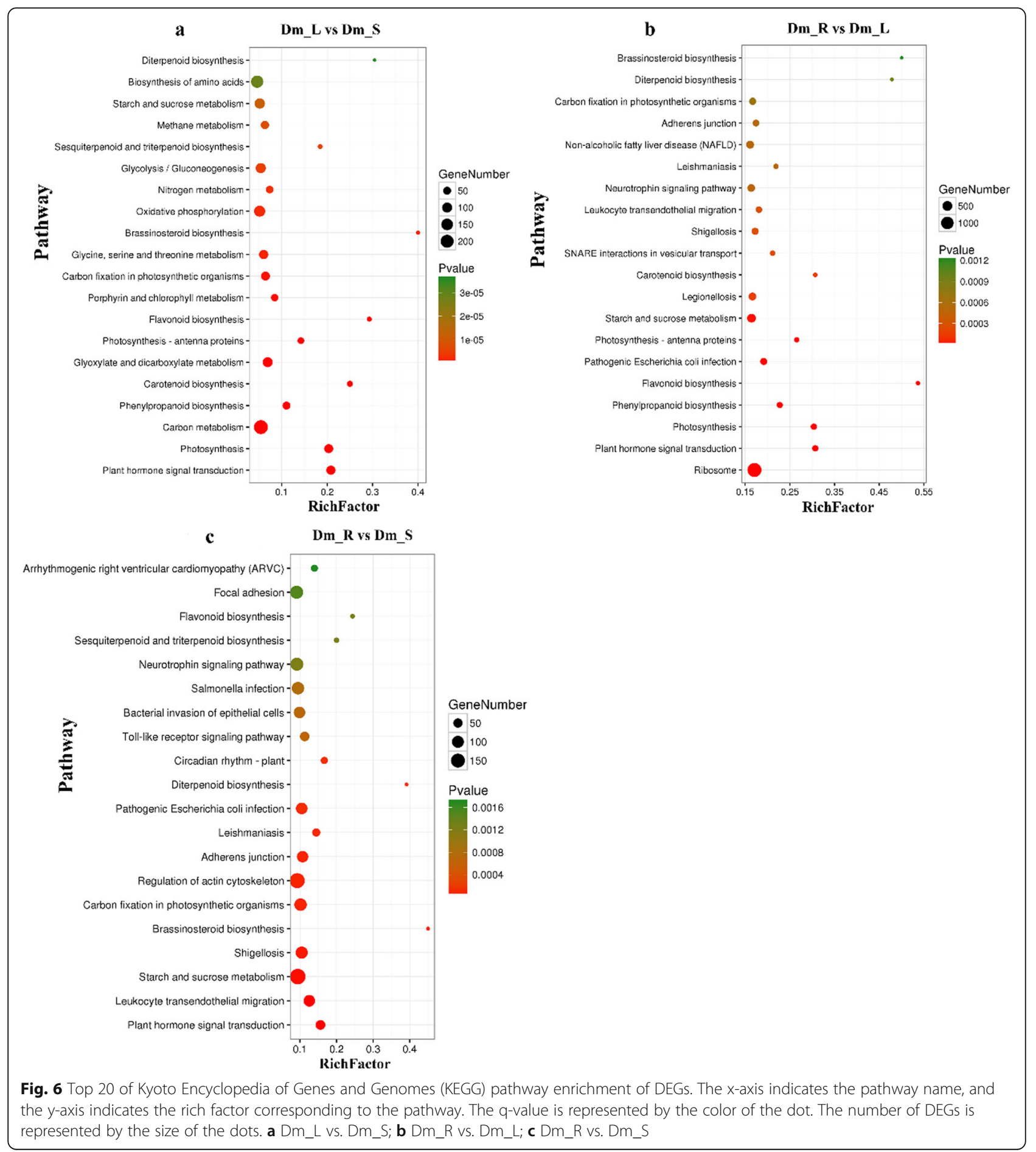

(FucTs) of DEGs, only one down-regulated DEG was identified between the Dm_R vs. Dm_L comparison and no DEGs were found in the other two comparisons. In xylosyltransferases (XTs) of DEGs, a total of two up-regulated DEGs were identified between Dm_L vs. Dm_S and two down-regulated DEGs were found between Dm_R vs. Dm_ L comparison (Fig. 8).
Cellulose is the most abundant organic matter in the biosphere and also is the structural polysaccharide of plants, the major component of their cell walls. The $D$. moniliforme cellulose synthase superfamily is divided into one CesA (cellulose synthase) family and nine Csl (cellulose synthase-like) families. Genes related to the cellulose synthase superfamily were involved in the 
Table 2 The pathways and number of unigenes related to secondary metabolites pathway

\begin{tabular}{|c|c|c|}
\hline Biosynthesis of secondary metabolic pathway & Pathway ID & Genes with pathway annotation $(152,667)$ \\
\hline Anthocyanin biosynthesis & ko00942 & $4(0.002 \%)$ \\
\hline Betalain biosynthesis & ko00965 & $98(0.064 \%)$ \\
\hline Brassinosteroid biosynthesis & ko00905 & $51(0.033 \%)$ \\
\hline Carotenoid biosynthesis & ko00906 & $168(0.110 \%)$ \\
\hline Caffeine metabolism & ko00232 & $92(0.060 \%)$ \\
\hline Cyanoamino acid metabolism & ko00460 & $600(0.393 \%)$ \\
\hline Diterpenoid biosynthesis & ko00904 & $76(0.050 \%)$ \\
\hline Flavone and flavonol biosynthesis & ko00944 & $22(0.014 \%)$ \\
\hline Flavonoid biosynthesis & ko00941 & $90(0.059 \%)$ \\
\hline Isoquinoline alkaloid biosynthesis & ko00950 & $385(0.252 \%)$ \\
\hline Monoterpenoid biosynthesis & ko00902 & $9(0.006 \%)$ \\
\hline Nicotinate and nicotinamide metabolism & ko00760 & $387(0.254 \%)$ \\
\hline Phenylpropanoid biosynthesis & ko00940 & $698(0.457 \%)$ \\
\hline Steroid biosynthesis & ko00100 & $626(0.410 \%)$ \\
\hline Terpenoid backbone biosynthesis & ko00900 & $598(0.392 \%)$ \\
\hline Tropane, piperidine and pyridine alkaloid biosynthesis & ko00960 & $292(0.191 \%)$ \\
\hline Monobactam biosynthesis & ko00261 & $168(0.110 \%)$ \\
\hline Streptomycin biosynthesis & ko00521 & $319(0.209 \%)$ \\
\hline Novobiocin biosynthesis & ko00401 & $64(0.042 \%)$ \\
\hline Aflatoxin biosynthesis & ko00254 & $104(0.068 \%)$ \\
\hline
\end{tabular}

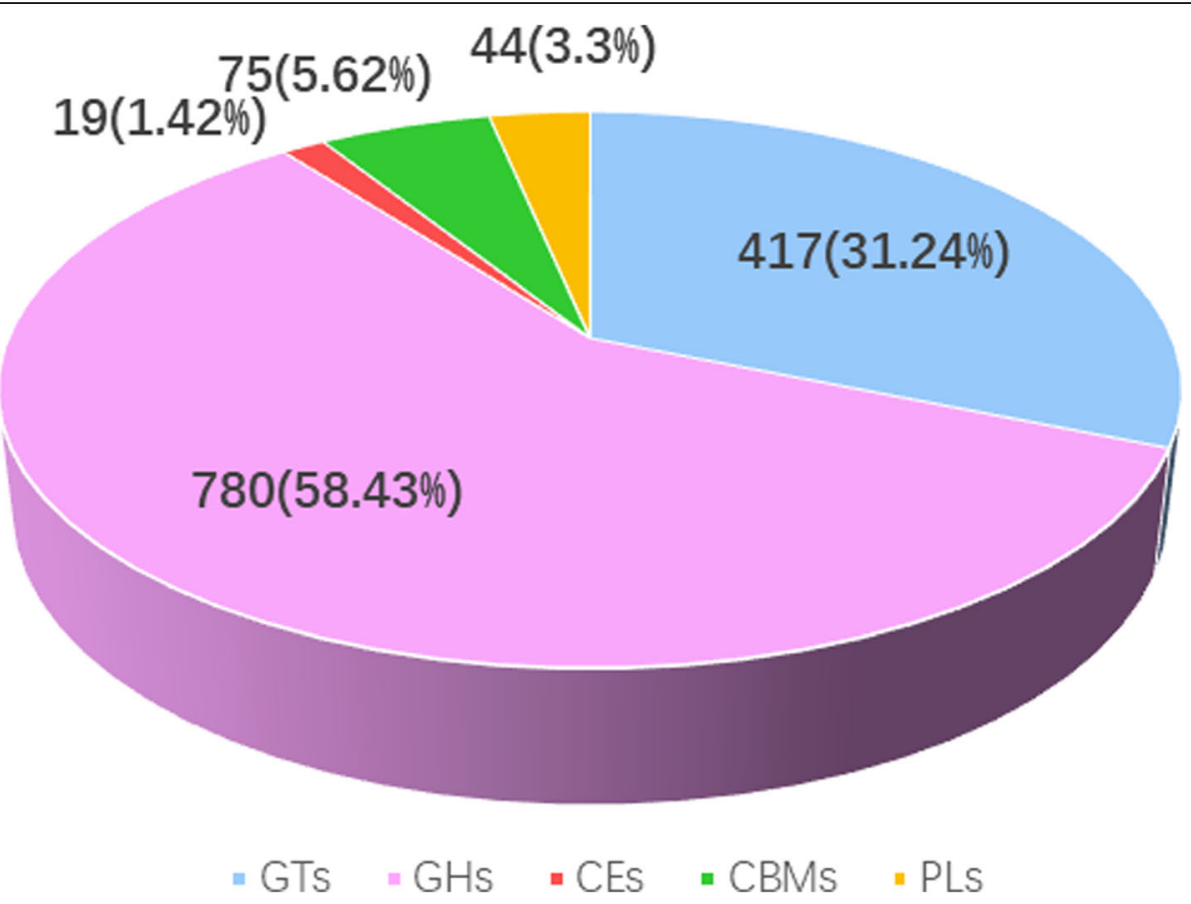

Fig. 7 The classification and number of carbohydrate-active enzyme families in D.moniliforme unigenes. GT, Glycosyltransferase; GH, Glycoside Hydrolase; CE, Carbohydrate Esterase; CBM, Carbohydrate-Binding Module; PL, Polysaccharide Lyase 
Table 3 The category and number of GT families in the DEGs database

\begin{tabular}{llll}
\hline Family & $\begin{array}{l}\text { Number of DEGs } \\
\text { (Dm_L vs. Dm_S) }\end{array}$ & $\begin{array}{l}\text { Number of DEGs } \\
(\text { Dm_R vs. Dm_L) }\end{array}$ & $\begin{array}{l}\text { Number of DEGs } \\
\text { (Dm_R vs. Dm_S) }\end{array}$ \\
\hline GT1 & 12 & 24 & 15 \\
GT2 & 6 & 15 & 9 \\
GT5 & 1 & 0 & 1 \\
GT15 & 0 & 1 & 1 \\
GT20 & 0 & 4 & 4 \\
GT22 & 0 & 2 & 2 \\
GT31 & 0 & 1 & 1 \\
GT34 & 0 & 1 & 1 \\
GT35 & 0 & 2 & 2 \\
GT41 & 0 & 1 & 1 \\
GT48 & 0 & 4 & 3 \\
GT62 & 0 & 1 & 0 \\
GT66 & 0 & 1 & 0 \\
Total & 19 & 57 & 40 \\
\hline
\end{tabular}

synthesis of mannan polysaccharides (Liepman et al., 2005). We identified 35 putative unigenes for CesA in $D$. moniliforme, which were classified into six families, CesA, CslC, CslD, CslE, CslG, CslH. Among 35 CesArelated genes, 21 genes showed differential expression in Fig. 9. These CesA-related DEGs were divided into five families, CesA, CslC, CslD, CslE and CslG with 8 and 5 DEGs belonging to CesA and CslD families, respectively.

\section{Analysis of putative genes and pathways involved in DMP biosynthesis}

Among 128 metabolic pathways detected in D. moniliforme, candidate genes related to starch and sucrose metabolic pathways were ranked in the top 20 as far as the number of involved genes was concerned (Table 4). To understand the biosynthesis of $D$. moniliforme polysaccharides (DMPs), the unigenes involved in the biosynthesis of starch and other related polymers, as well as those related to carbon assimilation in the tissues of stem, root and leaf, were annotated. Based on the KEGG database, we determined the key enzyme genes involved in these pathways (Table 3). The largest number of unigenes (17 unigenes) were identified as uridinediphosphate glucose pyrophosphorylase (galU) encoding genes; and the second largest number of unigenes (14) were annotated as hexokinase (HK) encoding genes; while the third largest number of unigenes (11) were annotated as phosphoglucomutase (pgm) encoding genes.

In plants, UDP-glucose pyrophosphorylase (UGPase) catalyzes the formation of uridine diphosphate glucose (UDP-Glu), a key precursor of nucleotide-diphosphosugar (NDP-sugar) formed from Glucose-1-phosphate (Glc-1P). Plant polysaccharides are formed by the active

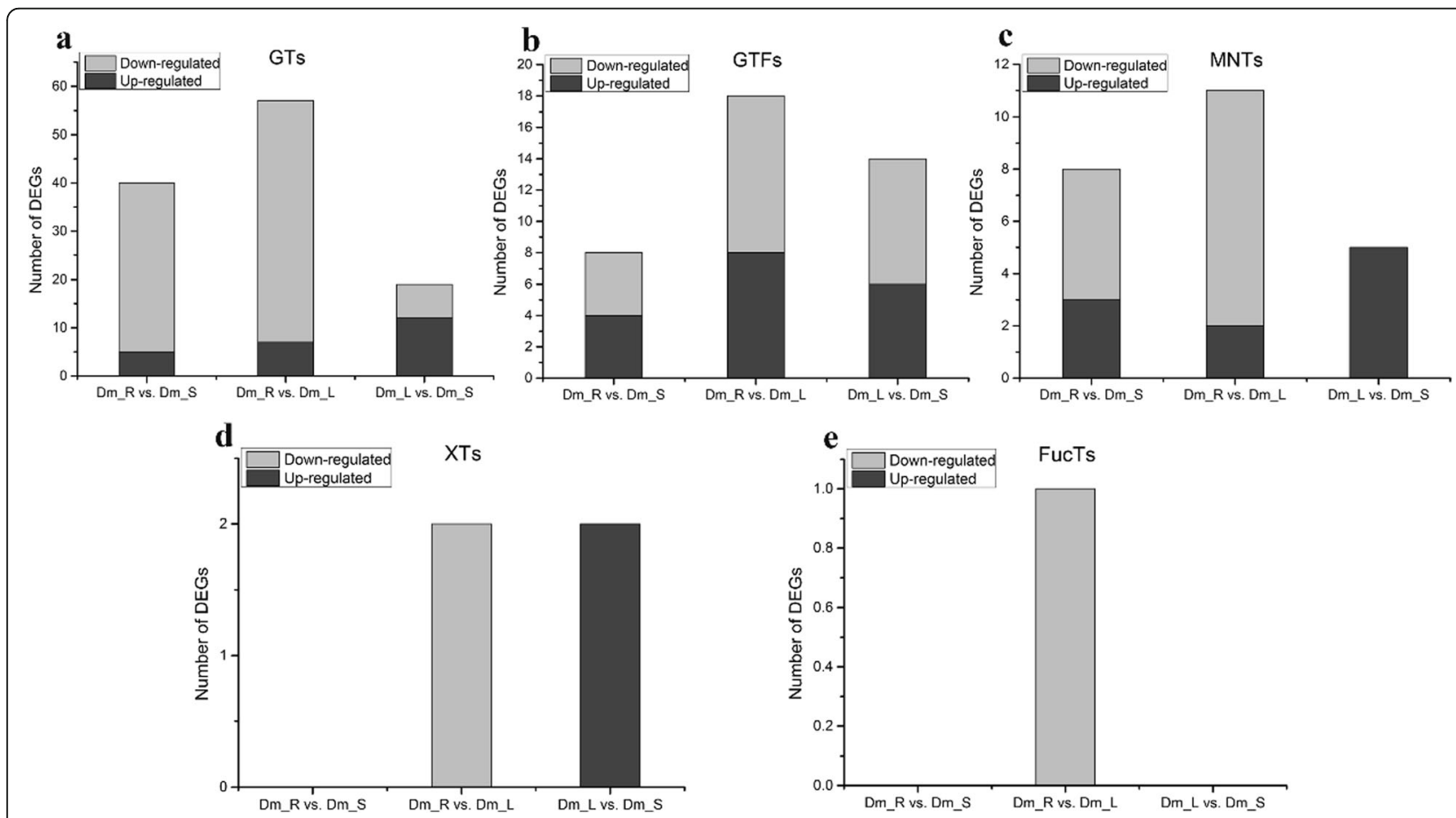

Fig. 8 Analysis of differentially expressed (DEGs) related to polysaccharide. The number of up-regulated and down-regulated between Dm_L vs. Dm_S, Dm_R vs. Dm_L, Dm_R vs. Dm_S are summarized. a Glycosyltransferases; b Glucosyltransferases; c Mannosyltransferases; d Xylosyltransferases; e Fucosyltransferases 


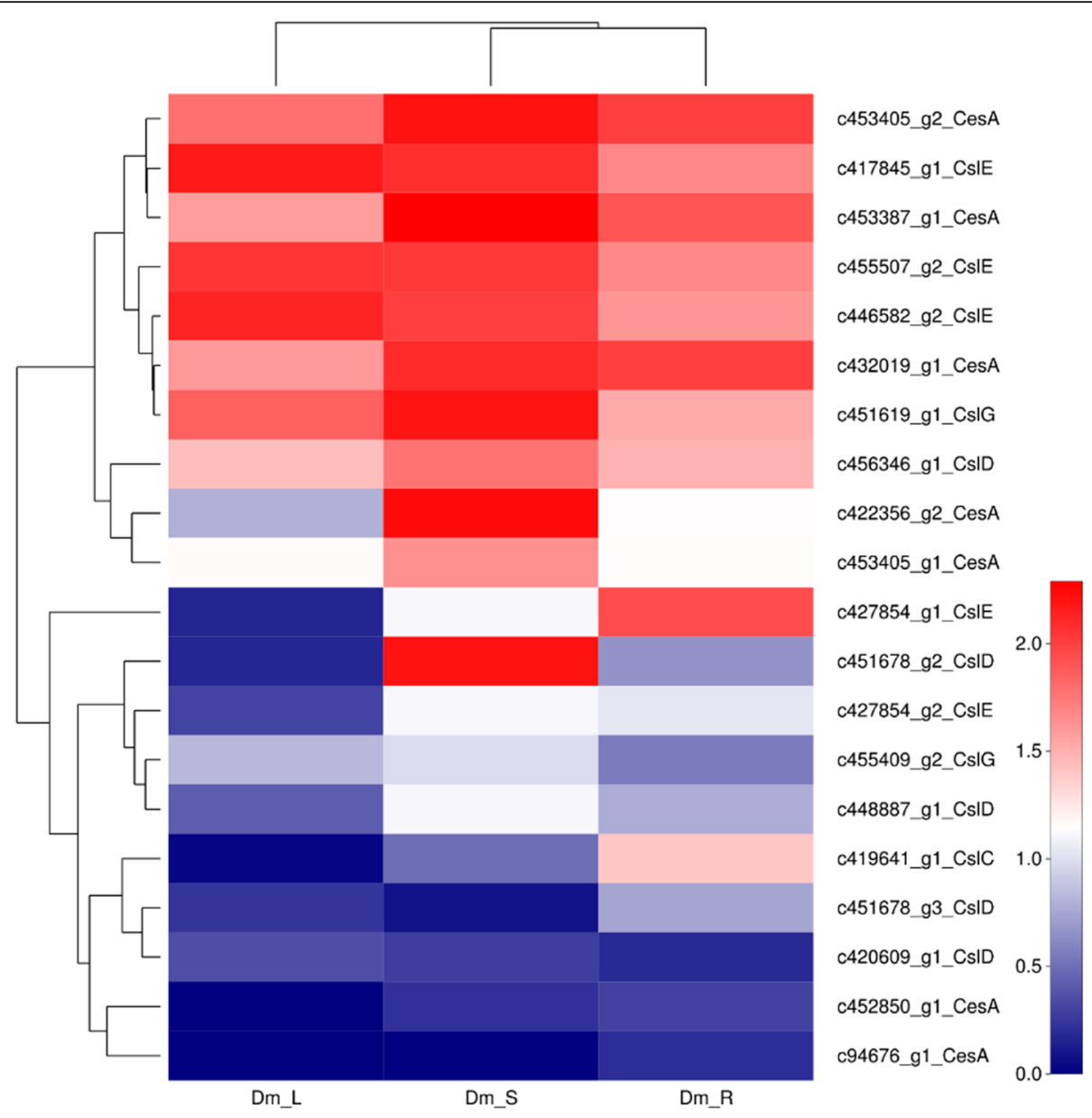

Fig. 9 The heatmap of DEGs involved in cellulose synthase superfamily. Red indicates high expression genes, while green indicates low expression genes. Color from red to green indicate that $\log 10($ FPKM +1$)$ gradually changes from big to small

NDP-sugar precursors, which are added to the residues of polysaccharides and glycoconjugates by the action of various glycosyltransferases (GTs) [31, 32]. The composition of cell walls is determined in part by the availability of NDP-sugars to form different types of wall polymers [33]. Since all polysaccharides are synthesized from activated NDP-sugars, the biosynthetic pathway of DMPs was inferred and predicted (Fig. 10).

Previous studies indicated that DMPs are formed by the polymerization of NDP-sugars such as UDP-galactose (UDP-Gal), UDP-D-xylose (UDP-D-Xyl), UDP-L-arabinose (UDP-L-Ara), UDP-Glucose (UDP-Glc), UDP-rhamnose (UDP-Rha), GDP-mannose (GDP-Man) and GDP-fucose (GDP-Fuc) [34, 35]. DMP biosynthesis can be divided into three main stages. First, sucrose is converted to Glc-6P and Fru-6P, leading to the formation of UDP-Glc and GDPMan from Glc-6P and Fru-6P, which are then converted in the second step into other NDP-sugars. Many enzymes play important roles in these processes, such as sacA [36], which converts sucrose to Glc-6P and Fructose, and pgm that isomerizes Glc-6P to Glc-1P [37]. HK [38] and scrK [39] also take part in the biosynthesis of Fru-6P. Secondly, UDP-Glc is derived from Glc-1P immediately [40], and Fru-6P is converted to GDP-Man indirectly [41]. Based on UDP-Glc and GDP-Man, other NDP sugars are further converted through the action of NDP-sugar interconversion enzymes (NSEs) [42], such as GALE, UGE, UXE and GMDS. Finally, the active monosaccharide units, NDPsugars, are added to the sugar residues of various polysaccharides and glycoconjugates by the action of various GTs.

\section{Transcription factors involved in $D$. moniliforme transcriptome dataset}

TFs have been implicated in a variety of developmental and physiological roles in plants. By comparison with the TFs from the iTAK database (http://itak.feilab.net/cgi-bin/ itak/index.cgi). These TFs belong to 66 known TF families (Table 5), the most abundant being the $\mathrm{C} 2 \mathrm{H} 2$ family,including 1158 unigenes. All these TFs have been identified as positive or negative regulators in the biosynthesis of secondary metabolites in other plants [43]. Previous genetic and molecular studies have revealed that NAC and 
Table 4 The number of DEGs involved in the biosynthesis of starch and sucrose in Dendrobium moniliforme

\begin{tabular}{|c|c|c|c|c|c|}
\hline Enzyme Code & Enzyme Name & Abbreviation & $\begin{array}{l}\text { Number of DEGs } \\
\text { (Dm_L vs. Dm_S) }\end{array}$ & $\begin{array}{l}\text { Number of DEGs } \\
\text { (Dm_R vs. Dm_L) }\end{array}$ & $\begin{array}{l}\text { Number of DEGs } \\
\text { (Dm_R vs. Dm_S) }\end{array}$ \\
\hline 3.2.1.26 & $\beta$-fructofuranosidase & sacA & 1 & 2 & 2 \\
\hline 5.4.2.2 & Phosphoglucomutase & pgm & 3 & 8 & 7 \\
\hline 2.7.7.9 & Uridine-diphosphate glucose pyrophosphorylase & galU & 4 & 15 & 10 \\
\hline 5.1.3.2 & UDP-glucose 4-epimerase & GALE & 3 & 8 & 4 \\
\hline 5.1.3.6 & UDP-glucuronate 4-epimerase & UGE & 3 & 2 & 1 \\
\hline 5.1.3.5 & UDP-arabinose 4-epimerase & UXE & 2 & 1 & 0 \\
\hline 5.3.1.9 & Glucose-6-phosphate isomerase & GPI & 2 & 9 & 6 \\
\hline 2.7.1.1 & Hexokinase & HK & 3 & 14 & 3 \\
\hline 2.7.1.4 & Fructokinase & scrk & 1 & 0 & 0 \\
\hline 5.3.1.8 & Mannose-6-phosphate isomerase & MPI & 3 & 3 & 3 \\
\hline 5.4.2.8 & Phosphomannomutase & PMM & 1 & 2 & 2 \\
\hline 2.7.7.13 & Mannose-1-phosphate guanylyltransferase & GMPP & 4 & 6 & 6 \\
\hline 4.2.1.47 & GDP-mannose 4,6-dehydratase & GMDS & 1 & 4 & 2 \\
\hline
\end{tabular}

MYB families, control secondary cell wall thickening in fibres, vessels, and anthers $[44,45]$ and control cellulose biosynthesis and assembling in cell walls [46]. We identified 133 MYB TFs and 90 NAC TFs in our transcriptome. MYB75 forms functional complexes to regulate secondary cell wall deposition and to integrate the metabolic flux through the lignin, flavonoid, and polysaccharide pathways in Arabidopsis [47]. SUSIBA2, a WRKY TF participated in sugar signaling by binding to the sugar-responsive elements of the iso1 promoter [48]. In our study, we discovered 88 WRKY TFs in the dataset. Through differential expression analysis, it was found that there were 30 differentially expressed MYB in roots, stems and leaves, 15 in NAC and 10 in WRKY.

\section{Real-time quantitative RT-PCR verification}

To validate changes in gene expression patterns, we selected two key enzyme-encoding genes associated with

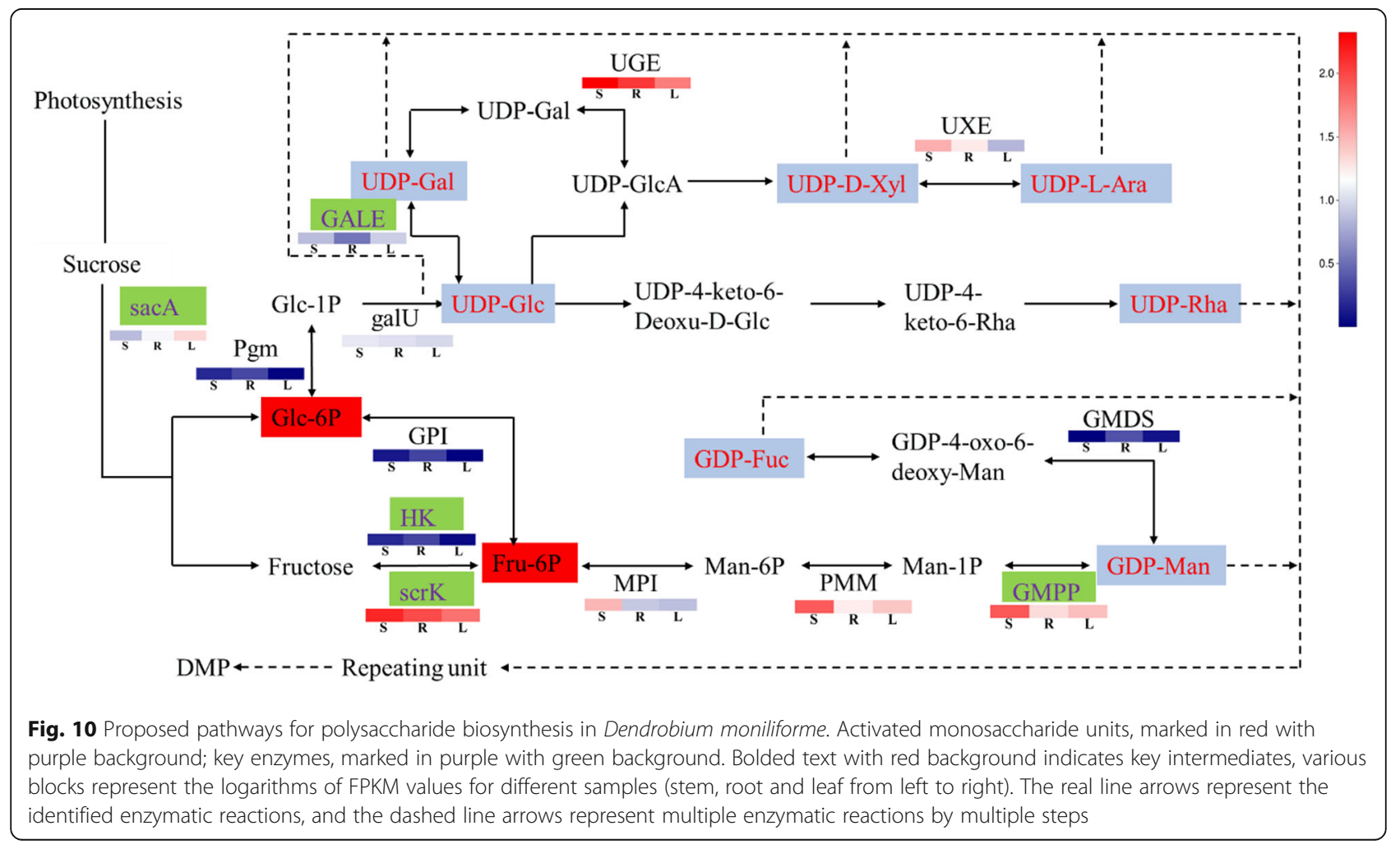


Table 5 Transcription factor families identified in the D. moniliforme transcriptome dataset

\begin{tabular}{llll}
\hline $\begin{array}{l}\text { Putative } \\
\text { transcription } \\
\text { factor family }\end{array}$ & $\begin{array}{l}\text { Number of gene } \\
\text { expressed in } \\
\text { transcriptome }\end{array}$ & $\begin{array}{l}\text { Putative } \\
\text { transcription } \\
\text { factor family }\end{array}$ & $\begin{array}{l}\text { Number of gene } \\
\text { expressed in } \\
\text { transcriptome }\end{array}$ \\
\hline AP2/ERF-ERF & 118 & LIM & 47 \\
B3 & 35 & LOB & 24 \\
bHLH & 221 & MADS-M-type & 62 \\
bZIP & 450 & MYB & 133 \\
C2C2-YABBY & 25 & MYB-related & 323 \\
C2C2-Dof & 41 & NAC & 90 \\
C2C2-GATA & 140 & NF-YB & 32 \\
C2H2 & 1158 & NF-YC & 51 \\
C3H & 476 & RWP-RK & 35 \\
E2F-DP & 25 & Trihelix & 32 \\
FAR1 & 50 & WRKY & 88 \\
GARP-G2-like & 77 & zf-HD & 13 \\
GRAS & 55 & zn-clus & 696 \\
HB-other & 131 & others & 423 \\
HSF & 108 & & \\
\hline
\end{tabular}

polysaccharide biosynthesis, including sucrose synthase (Susy) (c456394_g1, c435363_g4, c435363_g2, c449452_ g3) and sucrose phosphate synthase (SPS) (c448416_g1, c452034_g2, c452034_g1), and we examined these, using qRT-PCR at the transcriptional level. Primers and sequences are shown in Additional file 1: Table S1. The quantitative expression of DEGs is shown in Fig. 11a-g. Among all DEGs, the expression patterns of genes showed that the root samples had the lowest expression level of two key enzyme-encoding genes. However, we found that the two genes each accounted for half of the high expression in leaves and stems. The expression patterns of 2 DEGs were consistent with the transcriptome data $\left(R^{2}=0.91431, p\right.$-value $\left.=6.81571 \mathrm{E}-9\right)$. These results indicate that our transcriptomic analysis was highly reproducible and reliable (Fig. 11h).

\section{Discussion}

To date, this is the first time de novo sequencing of $D$. moniliforme has been conducted that has utilized the Illumina HiSeq platform. The sequencing produced $119.89 \mathrm{~Gb}$ clean data and 562,480 unigenes after assembly. The unigenes had the average distribution length of $703.77 \mathrm{bp}$, which is shorter than that from $D$. officinale (728 bp). We aligned to 169,702 unigenes $(48.99 \%)$ by BLASTX in the Nr database, but in proximal species- $D$. officinale, 70,146 unigenes showed significant similarity to known proteins in the $\mathrm{Nr}$ database. Our annotated unigene numbers are almost 2.5 fold greater than the unigene numbers from a previous study that focused on three different tissues [28]. There were 37,245 unigenes assigned to KOG database for functional prediction and classification. Our annotated unigene numbers are 1.4 fold fewer than the unigene numbers in $D$. officinale [28]. This information provides more adequate resources to study Dendrobium species.

KEGG is a major public pathway-related database that is able to analyze a gene product during a metabolic process and related gene function incellular processes [49]. We annotated 104,684 unigenes in the KEGG database. They were assigned to 503 KEGG pathways; among them, 20 KEGG pathways related to secondary metabolites were identified in $D$. moniliforme. There are many secondary metabolites and their chemical structures are different. These secondary metabolites are mainly formed in the plant by the phenylpropanoid metabolic pathway, isoprene metabolic pathway and the alkaloid synthesis pathway [50]. The biosynthesis of phenylpropanoids occupies an important position in most plants, such as tea plants [51], grapes [52], Polygonum minus [53], and D. moniliforme is the same to them. This result is consistent with most other studies. However, in Dendrobium, polysaccharides are the most important active ingredients. In this study, we therefore focus on the synthesis of $D$. moniliforme polysaccharides.

Polysaccharides are the main active constituents of $D$. moniliforme, which have the positive effects on human health. Research on D. moniliforme polysaccharides has been of great interest, especially studies on their content, composition, and pharmacological effects [54]. However, there are few reports on polysaccharide biosynthesis pathways and genes of Dendrobium species. The biosynthesis of polysaccharides involves hundreds of different GTs. The latest CAZy (http://www.cazy.org/GlycosylTransferases.html) indicates that the GTs are classified into 105 families, of which 42 and 43 GT families belong to Arabidopsis thaliana and Oryza sativa, respectively, with a total of 463 and 574 GT genes. In this study, we identified 413 putative GTs in the $D$. moniliforme transcriptome database that were divided into 13 families. In Dm_L vs. Dm_S, there are 19 DEGs of glycosyltransferase genes, belonging to 3 families (GT1, GT2 and GT5). In Dm_R vs. Dm_L, the largest number of DEGs was 57, which belong to 12 families. Compared with the other two comparisons, Dm_R vs. Dm_S has an intermediate number of 40 DEGs of glycosyltransferase genes, belonging to 10 families (Table 4). As can be seen from the above results, there are significant differences in the number of genes differentially expressed in aerial parts (leaf and stem) and root of D. moniliforme, but the differences in leaf and stem of aboveground parts are not significant. The stem of Dendrobium is the medicinal part. The medicinal ingredients all accumulate in the aerial parts, while the roots, which absorb nutrients from the matrix, transfer the nutrients to the aerial parts, so 

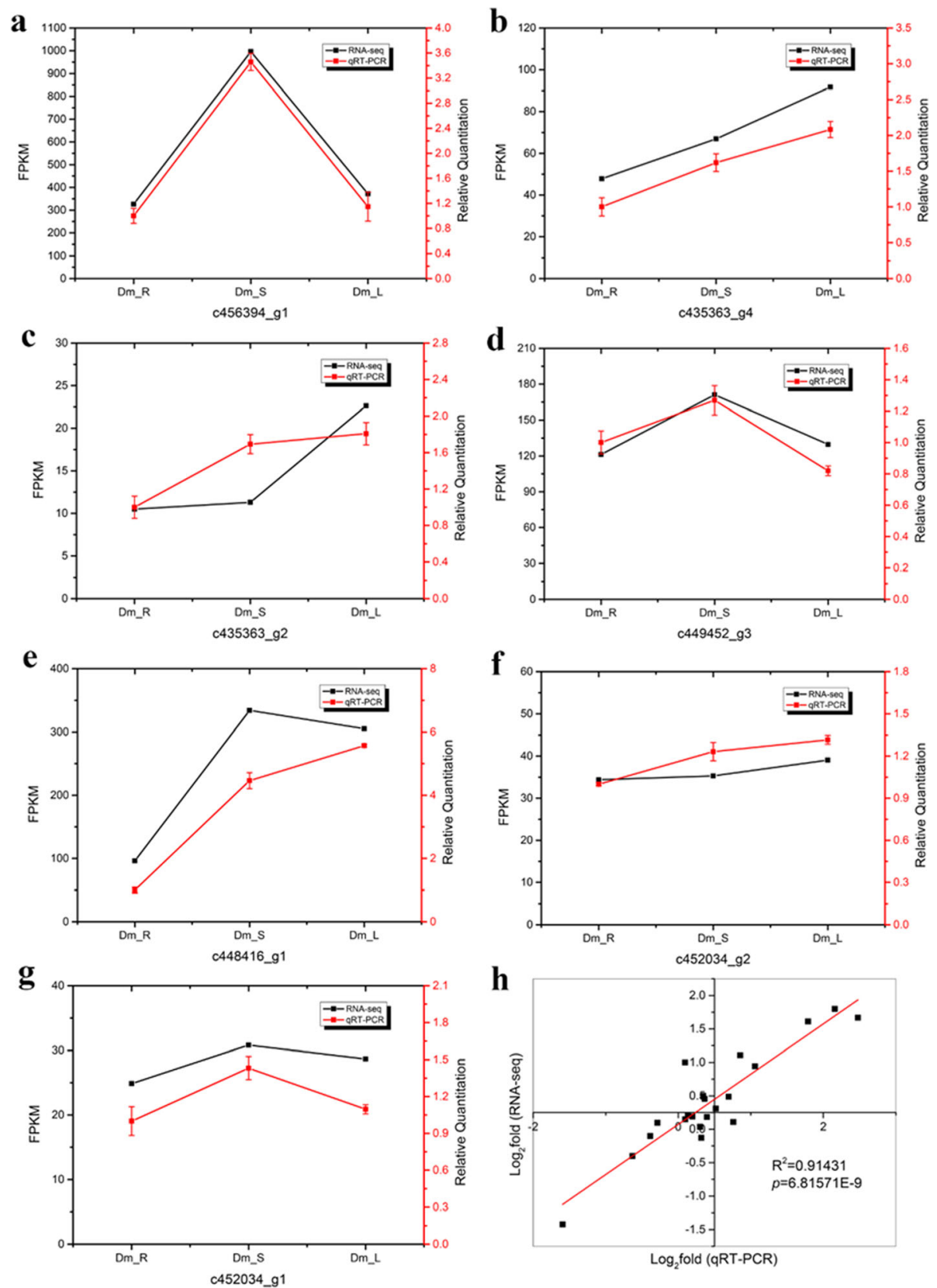

Fig. 11 qRT-PCR verification compared with expression profiles of DEGs. The real-time RT-PCR results were highly in agreement with the RNA-seq data. The $x$-axis represents the tissues of root, stem and leaf. The $y$-axis represents log (foldchange). The title means unigene ID. (a-g) represent the expression patterns of two key enzyme-encoding genes: a-d Susy; e-g SPS. Correlation analysis is shown in (h)

the content of the medicinal ingredients accumulated in the roots is not high. The GT1 family has the largest number among all three comparisons. It is a major GT family in plants and is also known as UDP glycosyltransferase (UGT) [55]. The UGT family, has about 120 UGTs in Arabidopsis, including eight distinct pseudogenes [56]. The second group consists of the GT2, GT20 and GT48 families, each accounting for approximately $10 \%$ of the genes. Glycosyltransferases, a multigene family that is widely involved in multiple metabolic pathways, need to respond to various changes in plant growth, the developmental environment, and other biological processes. Therefore, subsequent research on plant glycosyltransferases will have important scientific significance.

The family of mannans is the most wide spread group of polysaccharides in higher plants [57]. Cellulose synthase (CesA) superfamily genes were involved in the biosynthesis of mannan polysaccharides [58]. Cellulose is a 
major component of primary and secondary walls of plants and is synthesized from a rose-like protein complex of the CesA subunit, which consists of six rose-like subunits forming a larger rosette protein complex [59]. CesAs belongs to the glycosyltransferase-2 (GT-2) superfamily, which is classified into one cellulose synthase (CesA) family and nine cellulose synthase-like (Csl) families. The Csl families were divided into CslA/B/C/D/E/ F/G/H/J. In 1996, Pear et al. identified the first CesA gene in higher plants, GhCesA1 from Gossypium hirsutum [60]. The University of Michigan cloned PtrCesA1, the first cellulose synthase gene in tree wood from Populus tremuloides, a cellulose synthase gene specifically expressed in the xylem, and which is involved in the formation of secondary walls [61]. After that, 10 AtCesA from Arabidopsis thaliana, 18 PtriCesA from Populus trichocarpa and some CesA genes from Populus tremuloides and Eucalyptus camaldulensis were identified [62-64]. There are also many studies that have demonstrated that the CesA and CslA family are involved in the biosynthesis of mannan polysaccharides in many plant species. A $\beta$-mannan synthase (ManS) gene belonging to the CslA family from Cyamopsis tetragonolobus, creates the $\beta-1, \quad 4-m a n n a n$ backbone of galactomannan [65]. The CslA proteins from Arabidopsis is capable of producing $\beta$-mannan polysaccharides [66]. The research of these genes has laid a solid foundation for the resolution of other plant cellulose synthesis mechanisms. We identified 35 putative unigenes for CesA in the D. moniliforme RNA-seq data, which were classified into six families, CesA, CslC, CslD, CslE, CslG, and $\mathrm{CslH}$. CesA is expressed in all plant tissues and in different types of cells. However, the expression of different members of this gene family in the primary wall to secondary wall formation is different. Some studies have shown that the Csl families are involved in the biosynthesis of mannan polysaccharides. For instance, CslC subfamily members encode $\beta$-1,4-glucan synthase [67], CslD members have been implicated in cellulose synthesis [68]. while the CslG and $\mathrm{CslH}$ subfamily members are involved in the biosynthesis of $\beta-(1,3 ; 1,4)$-D-glucan $[69,70]$. Based on the transcriptome database, the expression of $\mathrm{CslG}$ and $\mathrm{CslH}$ in the stem is higher than in other tissues, which might encode some enzyme responsible for the synthesis of mannan polysaccharides in $D$. moniliforme.

Polysaccharide is the decisive factor in the quality of $D$. moniliforme. Sucrose also provides the substrate for the accumulation of polysaccharides. Therefore, we selected Sucrose Synthase (Susy) and Sucrose Phosphate Synthase (SPS), which are closely related to sucrose, for quantitative RT-PCR verification. SPS is one of the key enzymes that catalyze the synthesis of sucrose in the cytoplasm and are necessary for sucrose to enter various metabolic pathways. In a study of the tomato fruit, N'tchobo et al. considered SPS to play an important role in increasing tomato fruit sugar content and sucrose accumulation. When the SPS activity is higher, the soluble sugar content and sucrose content is higher [71]. Studies have shown that in sugarcane stems and leaves of sucrose content and SPS activity were positively correlated [72]. Most of Susy exists in the cytoplasm, which can catalyze the synthesis and decomposition of sucrose. It is speculated that SuSy activity directly affects the synthesis and metabolism of sucrose in plant organs and regulates the sucrose-starch metabolism of the organs [73, 74]. Tian et al. found that the accumulation of sucrose was mainly regulated by SuSy during the ripening of grape berries and strawberry fruits [75]. During the growth and development of grape berry, the expression level of SuSy is increased, which plays a leading role in the regulation of sugar metabolism in fruit [76]. In D. moniliforme, the expression of SPS and Susy in stems and leaves was significantly higher than that in roots, which were also affected by the regulation of SPS and Susy.

\section{Conclusions}

D. moniliforme is a famous Chinese herbal medicine and mainly distributed in tropical and subtropical regions. Polysaccharides are the main medicinal ingredients. To elucidate the molecular mechanisms of polysaccharides in D. moniliforme, we collected three different tissues and subjected them to high-throughput sequencing. A total of 562,480 unigenes were obtained in nine transcriptome libraries of $D$. moniliforme. In addition, 417 glycosyltransferases and 35 cellulose synthase genes were identified. Comparative analysis of the transcriptome in different tissues of $D$. moniliforme revealed a total of 35,159 DEGs that were mainly correlated with metabolic pathways and the biosynthesis of secondary metabolites. Our results provide understanding of the biosynthesis of DMPs at the molecular level in D. moniliforme. As the first report on the high-throughput sequencing of $D$. moniliforme, this study should provide novel insights into polysaccharidesrelated genes for $D$. moniliforme and should be a valuable molecular basis for study of Dendrobium spp.

\section{Methods}

\section{Plant materials}

D. moniliforme plants were artificially cultivated in the greenhouse of Anhui Tongjisheng Biotechnology Company, Lu'an, China. The original source was collected by the company from the wild after obtaining local permission. Seed germination and growth of protocorm-like bodies were cultured on half-strength Murashige and Skoog (MS) medium adding 6-BA $0.1 \mathrm{mg} \cdot \mathrm{L}^{-1}$, NAA 0.5 $\mathrm{mg} \cdot \mathrm{L}^{-1}$ and $1 \%$ additives $\left(30 \mathrm{~g} \cdot \mathrm{L}^{-1}\right.$ sucrose $+4 \mathrm{~g} \cdot \mathrm{L}^{-1}$ agar $+20 \%$ potato) under a $12 / 12 \mathrm{~h}$ light-dark cycle (approx. $30 \mu \mathrm{mol} \mathrm{m}{ }^{-2} \cdot \mathrm{S}^{-1}$ ) at $25 \pm 2{ }^{\circ} \mathrm{C}$. After the age of 6 months, the plants were transplanted into pots and placed in the 
greenhouse at a temperature of $25-27^{\circ} \mathrm{C}$ with a light/ dark cycle of $12 / 12 \mathrm{~h}$ and $60-70 \%$ relative humidity. Roots, stems and leaves of 2-year-old plants were harvested separately from three biological replicates in March, 2017. All of the D. moniliforme samples were frozen in liquid nitrogen and stored at $-80^{\circ} \mathrm{C}$ in an ultra-low temperature freezer for further processing. The voucher specimens were authenticated by Professor Jinchi Zhang and deposited at Jiangsu Province Key Laboratory of Soil and Water Conservation and Ecological Restoration in Nanjing Forestry University, Nanjing, China (Voucher number: 17C003).

\section{Determination of polysaccharide content}

Leaf, stem and root samples were collected from 2-yearold D. moniliforme at maturation stage. The phenolsulfuric acid method was applied to determine the polysaccharide contents in different tissues. The polysaccharide content was determined by using a glucose standard. Polysaccharides were extracted according to the method described by Chinese Pharmacopoeia (version 2010). Initially, samples $(0.3 \mathrm{~g})$ were precisely weighed, $200 \mathrm{ml}$ of water was added, and the samples heated and refluxed for $2 \mathrm{~h}$, filtered, then diluted to $250 \mathrm{ml}$. We accurately measured $5 \mathrm{ml}$ of the solution into a $50 \mathrm{ml}$ centrifuge tube, added $25 \mathrm{ml}$ of ethanol, shook and refrigerated this for $1 \mathrm{~h}$, centrifuged at $4000 \mathrm{r} \cdot \mathrm{min}^{-1}$ for $20 \mathrm{~min}$, discarded the supernatant, and centrifuged again with 20 $\mathrm{ml}$ of $80 \%$ ethanol (same as above). We repeated these steps 2 times, poured off the supernatant, and dissolved precipitate in heated water to a constant volume of 50 $\mathrm{ml} .1 \mathrm{ml}$ of sample solution was put in the test tube to which $1 \mathrm{ml}$ of $5 \%$ phenol solution was added. The solution was mixed thoroughly and $5 \mathrm{ml}$ of concentrated sulfuric acid was added, shaken and placed in a $75^{\circ} \mathrm{C}$ water bath for $20 \mathrm{~min}$. Afterwards, it was cooled until it was at room temperature, the absorbance was measured at 490 $\mathrm{nm}$ using UV-visible spectrophotometer with $1 \mathrm{ml}$ of water as a blank, and the test was performed in parallel three times.

RNA isolation, cDNA library construction and sequencing Total RNAs were extracted from these materials using OmniPlant RNA Kit (Cwbio, China). The purity of total RNA was analyzed using a Nanodrop 2000 Spectrophotometer (IMPLEN, USA). Total RNA quantity and quality were evaluated using NanoDrop 2000 spectrophotometer Agilent 2100 Bioanalyzer (Agilent Technologies, USA) and by agarose gel electrophoresis. According to the manufacturer's instructions, sequencing experiments were performed using the TruSeqTM RNA Sample Preparation Kit (Illumina, USA). In short, the protocol consists of the following steps: beads with oligo (dT) were used to isolate poly(A) mRNA from total RNA and cut into short fragments of 300 bp by adding fragmentation buffer. Taking these short fragments as templates, random hexamer primer was used to synthesize the first-strand cDNA, and then the second-strand cDNA was synthesized to form a stable double-stranded structure. The products were purified and enriched by PCR to create the final cDNA libraries. Finally, the library sequencing was performed on Illumina Hiseq 4000 platform (Illumina Inc., USA).

\section{De novo transcriptome assembly and unigenes annotation}

To get high-quality clean reads, we removed adaptercontaining, low-quality reads and poly- $\mathrm{N}$ from the raw data by using SeqPrep (https://github.com/jstjohn/SeqPrep) and Sickle (https://github.com/najoshi/sickle). Meanwhile, the calculation of Q20, Q30, GC-content and sequence duplication level were calculated based on the clean reads. All clean reads were assembled using Trinity software [77] based on the left.fq and right.fq, with the min_kmer_cov set as 2 by default and all other parameters set as their default.

Functional annotation of all the assembled unigenes was done by performing homology search against five major public databases. All the unigenes were annotated using BLASTx, with a cut-off E-value of $10^{-5}$ was taken as the threshold for significance. Five databases are as follows: $\mathrm{Nr}$ (NCBI non-redundant protein sequences, http://www.ncbi. nlm.nih.gov), Pfam (Protein family, http://pfam.sanger.ac. $\mathrm{uk} /$ ), String (Search tool for the retrieval of interacting genes, http://www.string-db.org/), Swiss-Prot (A manually annotated and reviewed protein sequence database, http:// www.uniprot.org) and KEGG (Kyoto encyclopedia of genes and genomes, http://www.genome. jp/kegg/).

\section{Identification of differentially expressed genes (DEGs)}

Gene expression level of each sample was estimated by RSEM version 1.2.15 [78]. The R Bioconductor package, edgeR [79] was used to identify the differentially expressed genes (DEGs) of two samples. The false discovery rate (FDR) criterion was used to calculate the threshold $P$-value in significance tests. To judge the significance of gene expression differences, we used FDR < 0.05 and $\mid \log _{2}$ (fold change) $\mid \geq 1$ as the threshold. GO enrichment analysis of DEGs was implemented by the Goatools [80]. For pathway enrichment analysis, KEGG enrichment analysis was performed using KOBAS version 2.0.12 [81].

\section{Quantitative real-time PCR (qRT-PCR)}

Studies have demonstrated that sucrose phosphate synthase (SPS) and sucrose synthase (Susy) are involved in sucrose metabolism [27]. These two genes were analyzed using qRT-PCR. Total RNA was extracted, as indicated above using new plant material, and three biological 
replicates were made. The specific primers for DEGs were designed by Oligo 7 software. The qRT-PCR was performed on an ABI 7500 Real-time PCR system (Applied Biosystems, USA) using SYBR Premix Ex Taq (Takara, Japan) according to the manufacturer's instructions. The expressions of SPS and Susy were normalized against Actin [25], an internal reference gene. All gene IDs and the primer sequences are listed in the Additional file 1: Table S1.

\section{Supplementary information}

Supplementary information accompanies this paper at https://doi.org/10. 1186/s12870-019-2138-7

Additional file 1: Figure S1: Sequence-length distribution of transcripts and unigenes assembled from Illumina. Figure S2. Venn diagram of all unigenes with annotations against five public databases. Figure S3. Functional gene ontology classification of unigenes. Figure S4. Kyoto Encyclopedia of Genes and Genomes (KEGG) pathway enrichment of DEGs.Table S1. Genes IDs and primers used in the quantitative real-time PCR (qRT-PCR) experiments.

Additional file 2. Differential expression genes between Dm_L and Dm_S

Additional file 3. Differential expression genes between Dm_R and Dm_L.

Additional file 4. Differential expression genes between Dm_R and Dm_S.

\section{Abbreviations}

CAZy: The carbohydrate-active enzyme; CBMs: Carbohydrate-binding modules; CEs: Carbohydrate esterases; CesA: Cellulose synthase; CesA: Cellulose synthase genes; Csl: Cellulose synthase-like.; DAG: Directed acyclic graph; DEGs: Differential expression genes; DMP: Dendrobium moniliforme polysaccharide; FDR: False discovery rate;

FucTs: Fucosyltransferases; GHs: Glycoside hydrolases; GTs: Glycosyltransferase genes; KEGG: Kyoto encyclopedia of genes and genomes; Nr: NCBI nonredundant protein sequences; Pfam: Protein family; PLs: Polysaccharide lyases; SPS: Sucrose phosphate synthase; String: Search tool for the retrieval of interacting genes; Susy: Sucrose synthase; XTs: Xylosyltransferases

\section{Acknowledgments}

We would thank Prof. Donald L. DeAngelis, from the Wetland and Aquatic Research Center, U.S. Geological Survey, for his valuable comments and suggestions that have greatly improved the quality of this manuscript.

\section{Authors' contributions}

Conception and design of the research: YY and JZ; acquisition of data: $J L$ and $\mathrm{XL}$; analysis and interpretation of data: MM; statistical analysis: JL; drafting the manuscript: YY and JZ; revision of manuscript for important intellectual content: JK. All authors read and approved the final manuscript.

\section{Funding}

This project was funded by the Priority Academic Program Development of Jiangsu Higher Education Institutions (PAPD), National Foundation of Forestry Science and Technology Popularization (Grant No. [2015]17), Major Fund for Natural Science of Jiangsu Higher Education Institutions (Grant No. 15KJA220004). The funders had no role in study design, data collection and analysis, decision to publish, or preparation of the manuscript.

\section{Availability of data and materials}

All sequence data have been deposited at the National Center for Biotechnology Information short read archive (SRA) under the accession number SRP139000.

\section{Ethics approval and consent to participate}

Dendrobium moniliforme used in this study were cultivated by Maoyun Yu's greenhouse from Anhui Tongjisheng Biotechnology Company, Lu'an, China. No permits were required for the collection of the samples.

\section{Consent for publication}

Not applicable.

\section{Competing interests}

The authors declare that they have no competing interests.

\section{Author details}

${ }^{1}$ Co-Innovation Center for Sustainable Forestry in Southern China, Nanjing Forestry University, Nanjing 210037, China. ${ }^{2}$ Jiangsu Province Key Laboratory of Soil and Water Conservation and Ecological Restoration, Nanjing Forestry University, Nanjing 210037, China. ${ }^{3}$ University of Miami, Coral Gables 33146, USA.

Received: 8 July 2019 Accepted: 14 November 2019

Published online: 27 November 2019

\section{References}

1. Wu X, Yuan J, Luo A, Chen Y, Fan Y. Drought stress and re-watering increase secondary metabolites and enzyme activity in dendrobium moniliforme. Ind Crop Prod. 2016;94:385-93.

2. Chin-Tung W, Keng-Shiang H, Chih-Hui Y, Yu-Chang C, Jiunn-Wang L, ChaoLin K, Chung-Li C, Shu-Fang L, Chang-Chi H, Hsin-Sheng T. Inhibitory effects of cultured Dendrobium tosaense on atopic dermatitis murine model. Int J Pharm. 2014;463(2):193-200

3. Juan L, Shunxiang L, Dan H, Xingbing Z, Guangxian C. Advances in the of resources, constituents and pharmacological effects of Dendrobium officinale. Sci Technol Rev. 2011;29(18):74-9.

4. Hua YF, Zhang M, Fu CX, Chen ZH, Chan GY. Structural characterization of a 2-O-acetylglucomannan from Dendrobium officinale stem. Carbohydr Res. 2004:339(13):2219-24

5. Chen $\mathrm{H}$, Zhang M, Xie B. Components and antioxidant activity of polysaccharide conjugate from green tea. Food Chem. 2005;90(1-2):17-21.

6. Hsieh YS, Chien C, Liao SK, Liao SF, Hung WT, Yang WB, Lin CC, Cheng TJ, Chang CC, Fang JM. Structure and bioactivity of the polysaccharides in medicinal plant Dendrobium huoshanense. Bioorg Med Chem. 2008;16(11): 6054.

7. Fan Y, He X, Zhou S, Luo A, He T, Chun Z. Composition analysis and antioxidant activity of polysaccharide from Dendrobium denneanum. Int J Biol Macromol. 2009:45(2):169.

8. Abougheangone $\mathrm{S}$, Nguemaona E, Boudjeko T, Driouich A. Plant cell wall polysaccharides: immunomodulators of the immune system and source of natural fibers; 2011.

9. Luo A, He X, Zhou S, Fan Y, He T, Chun Z In vitro antioxidant activities of a water-soluble polysaccharide derived from Dendrobium nobile Lindl. Extracts. Int J Biol Macromol. 2009:45(4):359-63.

10. He C, Zhang J, Liu X, Zeng S, Wu K, Yu Z, Wang X, Teixeira da Silva JA, Lin Z, Duan J. Identification of genes involved in biosynthesis of mannan polysaccharides in Dendrobium officinale by RNA-seq analysis. Plant Mol Biol. 2015;88(3):219-31.

11. Luo QL, Tang ZH, Zhang XF, Zhong YH, Yao SZ, Wang LS, Lin CW, Luo X. Chemical properties and antioxidant activity of a water-soluble polysaccharide from Dendrobium officinale. Int J Biol Macromol. 2016;89: 219-27.

12. Li XL, Xiao JJ, Zha XQ, Pan LH, Asghar MN, Luo JP. Structural identification and sulfated modification of an antiglycation Dendrobium huoshanense polysaccharide. Carbohydr Polym. 2014;106:247-54.

13. Pan L-H, Feng B-J, Wang J-H, Zha X-Q, Luo J-P. Structural characterization and anti-Glycation Activityin vitro of a water-soluble polysaccharide from Dendrobium Huoshanense. J Food Biochem. 2013:37(3):313-21.

14. Bouchez D, Höfte H. Functional genomics in plants. Plant Physiol. 1998; 118(3):725.

15. Colebatch G, Trevaskis B, Udvardi M. Functional genomics: tools of the trade. New Phytol. 2002:153(1):27-36

16. Sato S, Hirakawa H, Isobe S, Fukai E, Watanabe A, Kato M, Kawashima K, Minami C, Muraki A, Nakazaki N. Sequence analysis of the genome of an oilbearing tree, Jatropha curcas L. DNA Res. 2010;18(1):65. 
17. André S, Sun Y, Song J, Wu Q, Sun C, Li Y, Luo H, Chen S. Comparison of 454-ESTs from Huperzia serrata and Phlegmariurus carinatus reveals putative genes involved in lycopodium alkaloid biosynthesis and developmental regulation. BMC Plant Biol. 2010;10(1):209.

18. Qian J, Xu H, Song J, Xu J, Zhu Y, Chen S. Genome-wide analysis of simple sequence repeats in the model medicinal mushroom Ganoderma lucidum. Gene. 2013;512(2):331-6.

19. Molina C, Rotter B, Horres R, Udupa SM, Besser B, Bellarmino L, Baum M, Matsumura H, Terauchi R, Kahl G. SuperSAGE: the drought stress-responsive transcriptome of chickpea roots. BMC Genomics. 2008;9(1):553.

20. Zhao S, Tuan P, Li X, Kim Y, Kim H, Park C, Yang J, Li C, Park S. Identification of phenylpropanoid biosynthetic genes and phenylpropanoid accumulation by transcriptome analysis of Lycium chinense. BMC Genomics. 2013;14:802.

21. Gao W, Sun HX, Xiao H, Cui G, Hillwig ML, Jackson A, Wang X, Shen Y, Zhao $N$, Zhang $L$, et al. Combining metabolomics and transcriptomics to characterize tanshinone biosynthesis in Salvia miltiorrhiza. BMC Genomics. 2014;15:73.

22. Pollier J, Vanden Bossche R, Rischer $\mathrm{H}$, Goossens A. Selection and validation of reference genes for transcript normalization in gene expression studies in Catharanthus roseus. Plant Physiol Biochem. 2014;83:20-5.

23. Wu D, Austin RS, Zhou S, Brown D. The root transcriptome for north American ginseng assembled and profiled across seasonal development. BMC Genomics. 2013;14:564.

24. Shen C, Hong G, Chen H, Shi Y, Meng Y, Lu J, Feng S, Wang H. Identification and analysis of genes associated with the synthesis of bioactive constituents in Dendrobium officinale using RNA-Seq. Sci Rep. 2017;7(1):187.

25. Zhang J, He C, Wu K, Teixeira da Silva J, Zeng S, Zhang X, Yu Z, Xia H, Duan J. Transcriptome analysis of Dendrobium officinale and its application to the identification of genes associated with polysaccharide synthesis. Front Plant Sci. 2016;7:5

26. Guo X, Li Y, Li C, Luo H, Wang L, Qian J, Luo X, Xiang L, Song J, Sun C, et al. Analysis of the Dendrobium officinale transcriptome reveals putative alkaloid biosynthetic genes and genetic markers. Gene. 2013;527(1):131-8.

27. Yan L, Wang X, Liu H, Tian Y, Lian J, Yang R, Hao S, Wang X, Yang S, Li Q, et al. The genome of Dendrobium officinale illuminates the biology of the important traditional Chinese orchid herb. Mol Plant. 2015;8(6):922-34

28. Meng Y, Yu D, Jie X, Lu J, Feng S, Shen C, Wang H. A transcriptome-wide, organ-specific regulatory map of Dendrobium officinale, an important traditional Chinese orchid herb. Sci Rep. 2016;6:18864.

29. Kutchan TM. Ecological arsenal and developmental dispatcher. The paradigm of secondary metabolism. Plant Physiol. 2001;125(1):58-60

30. Campbell JA, Davies GJ, Bulone V, Henrissat B. A classification of nucleotidediphospho-sugar glycosyltransferases based on amino acid sequence similarities. Biochem J. 1997;326(Pt 3):929.

31. Pauly M, Gille S, Liu L, Mansoori N, De SA, Schultink A, Xiong G. Hemicellulose biosynthesis. Planta. 2013;238(4):627.

32. Wang Y, Alonso AP, Wilkerson CG, Keegstra K. Deep EST profiling of developing fenugreek endosperm to investigate galactomannan biosynthesis and its regulation. Plant Mol Biol. 2012;79(3):243.

33. Nag A, Karpinets TV, Chang CH, Barpeled M. Enhancing a Pathway-Genome Database (PGDB) to capture subcellular localization of metabolites and enzymes: the nucleotide-sugar biosynthetic pathways of Populus trichocarpa. Database (Oxford). 2012;2012(4):bas013.

34. Yang $X$, Zhao Y, Ruan Y, Yang Y. Development and application of a capillary electrophoretic method for the composition analysis of a typical heteropolysaccharide from Codonopsis pilosula NANNF. Biol Pharm Bull. 2008;31(10):1860-5.

35. YaJun Z, LiXia Z, JingFeng Y, ZhongYan L. Structure analysis of watersoluble polysaccharide CPPS3 isolated from Codonopsis pilosula. Fitoterapia. 2010;81(3):157.

36. Xie Y, Zhou H, Liu C, Zhang J, Li N, Zhao Z, Sun G, Zhong Y. A molasses habitat-derived fungus Aspergillus tubingensis XG21 with high $\beta$ fructofuranosidase activity and its potential use for fructooligosaccharides production. AMB Express. 2017;7(1):128.

37. Uematsu K, Suzuki N, Iwamae T, Inui M, Yukawa H. Expression of Arabidopsis plastidial phosphoglucomutase in tobacco stimulates photosynthetic carbon flow into starch synthesis. J Plant Physiol. 2012; 169(15):1454-62.

38. Zhang $Y$, Zhen $L$, Tan $X$, Li L, Wang $X$. The involvement of hexokinase in the coordinated regulation of glucose and gibberellin on cell wall invertase and sucrose synthesis in grape berry. Mol Biol Rep. 2014;41(12):7899-910.
39. Perezcenci M, Salerno GL. Functional characterization of Synechococcus amylosucrase and fructokinase encoding genes discovers two novel actors on the stage of cyanobacterial sucrose metabolism. Plant Sci. 2014;224(13):95-102.

40. Park J, Ishimizu T, Suwabe K, Sudo K, Masuko H, Hakozaki H, Nou I, Suzuki G, Watanabe M. UDP-glucose Pyrophosphorylase is rate limiting in vegetative and reproductive phases in Arabidopsis thaliana. Plant Cell Physiol. 2010; 51(6):981.

41. Bachmann P, Zetsche K. A close temporal and spatial correlation between cell growth, Cell Wall synthesis and the activity of enzymes of Mannan synthesis in Acetabularia mediterranea. Planta. 1979;145(4):331-7.

42. Yanbin Yin JH, Xiaogang G, Bar-Peled M, Xu Y. Evolution of plant nucleotide-sugar Interconversion enzymes. PLoS One. 2011;6(11):e27995.

43. Fits $L$, Van Der MJ. ORCA3, a jasmonate-responsive transcriptional regulator of plant primary and secondary metabolism. Science. 2000;289(5477):295-7.

44. Nobutaka M, Motoaki S, Kazuo S, Masaru OT. The NAC transcription factors NST1 and NST2 of Arabidopsis regulate secondary wall thickenings and are required for anther dehiscence. Plant Cell. 2005;17(11):2993-3006.

45. Caiyun Y, Zhengyao X, Jie S, Katie C, Gema VB, Wilson ZA. Arabidopsis MYB26/MALE STERILE35 regulates secondary thickening in the endothecium and is essential for anther dehiscence. Plant Cell. 2007;19(2):534-48.

46. Xie L, Yang C, Wang X. Brassinosteroids can regulate cellulose biosynthesis by controlling the expression of CESA genes in Arabidopsis. J Exp Bot. 2011; 62(13):4495-506.

47. Chaudhary P, Bahl A, Kumar A. MYB75 functions in regulation of secondary cell wall formation in the Arabidopsis inflorescence stem. Plant Physiol. 2010;154(3):1428-38.

48. Chuanxin S, Sara P, Helena O, Mats B, Staffan A, Christer J. A novel WRKY transcription factor, SUSIBA2, participates in sugar signaling in barley by binding to the sugar-responsive elements of the iso1 promoter. Plant Cell. 2003;15(9):2076-92.

49. Kanehisa M, Araki M, Goto S, Hattori M, Hirakawa M, Itoh M, Katayama T, Kawashima S, Okuda S, Tokimatsu T. KEGG for linking genomes to life and the environment. Nucleic Acids Res. 2008;36(Database issue):480-4.

50. Leach D. Function of Plant Secondary Metabolites and their Exploitation in Biotechnology, Annual Plant Reviews, Volume 3 : Edited by Michael Wink ( publishers: Sheffield \& CRC Press ). Biochem Syst Ecol. 2002;30(3):279.

51. Guo F, Guo Y, Wang P, Wang Y, Ni D. Transcriptional profiling of catechins biosynthesis genes during tea plant leaf development. Planta. 2017;246(6):1139-52.

52. Marè C, Aprile A, Roncaglia E, Tocci E, Corino LG, Bellis LD, Cattivelli L. Rootstock and soil induce transcriptome modulation of phenylpropanoid pathway in grape leaves. J Plant Interact. 2013;8(4):334-49.

53. Loke KK, Rahnamaietajadod R, Yeoh CC, Goh HH, Mohamedhussein ZA, Zainal Z, Ismail I, Noor NM. Transcriptome analysis of Polygonum minus reveals candidate genes involved in important secondary metabolic pathways of phenylpropanoids and flavonoids. Peerj. 2017;5(2):e2938.

54. Sun L, Zhang XD, Guo YY, Academy JG. Research Progress on chemical composition and pharmacological of MORI FRUCTUS. Ginseng Res. 2016;(2): 49-54 (in Chinese).

55. Weis M, Lim EK, Bruce NC, Bowles DJ. Engineering and kinetic characterisation of two glucosyltransferases from Arabidopsis thaliana. Biochimie. 2008;90(5):830-4.

56. Paquette S, Møller BL, Bak S. On the origin of family 1 plant glycosyltransferases. Phytochemistry. 2003;62(3):399.

57. Moreira LRS, Filho EXF. An overview of mannan structure and mannandegrading enzyme systems. Appl Microbiol Biotechnol. 2008;79(2):165-78.

58. Liepman AH, Wilkerson CG, Kenneth K. Expression of cellulose synthase-like (CSI) genes in insect cells reveals that CSIA family members encode mannan synthases. Proc Natl Acad Sci U S A. 2005;102(6):2221-6.

59. Mcfarlane HE, Döring A, Persson S. The cell biology of cellulose synthesis. Annu Rev Plant Biol. 2014;65(1):69.

60. Pear JR, Kawagoe Y, Schreckengost WE, Delmer DP, Stalker DM. Higher plants contain homologs of the bacterial celA genes encoding the catalytic subunit of cellulose synthase. Proc Natl Acad Sci U S A. 1996;93(22):12637-42.

61. Wu L, Joshi CP, Chiang VL. A xylem-specific cellulose synthase gene from aspen (Populus tremuloides) is responsive to mechanical stress. Plant $J$. 2000;22(6):495-502.

62. Suzuki S, Li L, Sun YH, Chiang VL. The cellulose synthase gene superfamily and biochemical functions of xylem-specific cellulose synthase-like genes in Populus trichocarpa. Plant Physiol. 2006;142(3):1233.

63. Richmond TA, Somerville CR. The cellulose synthase superfamily. Plant Physiol. 2000;124(2):495-8. 
64. Endler A, Persson S. Cellulose synthases and synthesis in Arabidopsis. Mol Plant. 2011;4(2):199-211.

65. Dhugga KS, Roberto B, Brad W, Kevin S, Jan H, Randhawa GS, Maureen D, Kinney AJ, Dwight T, Scott N. Guar seed beta-mannan synthase is a member of the cellulose synthase super gene family. Science. 2004; 303(5656):363-6.

66. Florence G, Barton CJ, Mortimer JC, Xiaolan Y, Zhinong Z, Miles GP, Jenny R, Liepman AH, Keith S, Paul D. Cell wall glucomannan in Arabidopsis is synthesised by CSLA glycosyltransferases, and influences the progression of embryogenesis. Plant J. 2010;60(3):527-38,

67. Cocuron JC, Lerouxel O, Drakakaki G, Alonso AP, Liepman AH, Keegstra K, Raikhel $\mathrm{N}$, Wilkerson CG. A gene from the cellulose synthase-like C family encodes a beta-1,4 glucan synthase. Proc Natl Acad Sci U S A. 2007;104(20):8550.

68. Galway ME, Eng RC, Schiefelbein JW, Wasteneys GO. Root hair-specific disruption of cellulose and xyloglucan in AtCSLD3 mutants, and factors affecting the post-rupture resumption of mutant root hair growth. Planta. 2011;233(5):985-99.

69. Shin T, Takahisa Y, Takuji T, Yoichi T, Yoshiaki I, Naoto H, Oscar L, Jobling SA. Functional characterization of barley betaglucanless mutants demonstrates a unique role for CsIF6 in (1,3;1,4)- $\beta$-D-glucan biosynthesis. J Exp Bot. 2012; 63(1):381-92.

70. Nemeth C, Freeman J, Jones HD, Sparks C, Pellny TK, Wilkinson MD, Dunwell J, Andersson AA, Aman P, Guillon F. Down-regulation of the CSLF6 gene results in decreased (1,3;1,4)-beta-D-glucan in endosperm of wheat. Plant Physiol. 2010;152(3):1209-18.

71. N'tchobo H, Dali N, Nguyen-Quoc B, Foyer CH, Yelle S. Starch synthesis in tomato remains constant throughout fruit development and is dependent on sucrose supply and sucrose synthase activity. J Exp Bot. 1999;50(338): 1457-63.

72. Grof CPL, Albertson PL, Bursle J, Perroux JM, Bonnett GD, Manners JM. Sucrose-phosphate synthase, a biochemical marker of high sucrose accumulation in sugarcane. Crop Sci. 2007;47(4):1530-9.

73. Angeles-Núñez JG, Tiessen A. Regulation of AtSUS2 and AtSUS3 by glucose and the transcription factor LEC2 in different tissues and at different stages of Arabidopsis seed development. Plant Mol Biol. 2012;78(4-5):377-92.

74. Jiang Y, Guo W, Zhu H, Ruan YL, Zhang T. Overexpression of GhSusA1 increases plant biomass and improves cotton fiber yield and quality. Plant Biotechnol J. 2012;10(3):301-12.

75. Tian $L$, Jia HF, Li CL, Fan PG, Xing Y, Shen YY. Sucrose accumulation during grape berry and strawberry fruit ripening is controlled predominantly by sucrose synthase activity. J Pomology Hortic Sci. 2012;87(6):661-7.

76. Wang $X Q$, Li LM, Yang PP, Gong CL. The role of hexokinases from grape berries (Vitis vinifera L.) in regulating the expression of cell wall invertase and sucrose synthase genes. Plant Cell Rep. 2014;33(2):337-47.

77. Grabherr MG, Haas BJ, Yassour M, Levin JZ, Thompson DA, Amit I, Adiconis $X$, Fan L, Raychowdhury R, Zeng Q. Full-length transcriptome assembly from RNA-Seq data without a reference genome. Nat Biotechnol. 2011;29(7):644.

78. Li B, Dewey CN. RSEM: accurate transcript quantification from RNA-Seq data with or without a reference genome. BMC Bioinformatics. 2011;12(1):323.

79. Robinson MD, Mccarthy DJ, Smyth GK. edgeR: a Bioconductor package for differential expression analysis of digital gene expression data. Bioinformatics. 2010;26(1):139.

80. Tang H, Flick P, Sato K, Ramirez F, Klopfenstein D, Mungall C, Yunes J, Pedersen B. GOATOOLS: tools for gene ontology; 2015.

81. Mao X, Cai T, Olyarchuk JG, Wei L. Automated genome annotation and pathway identification using the KEGG Orthology (KO) as a controlled vocabulary. Bioinformatics. 2005;21(19):3787.

\section{Publisher's Note}

Springer Nature remains neutral with regard to jurisdictional claims in published maps and institutional affiliations.

Ready to submit your research? Choose BMC and benefit from:

- fast, convenient online submission

- thorough peer review by experienced researchers in your field

- rapid publication on acceptance

- support for research data, including large and complex data types

- gold Open Access which fosters wider collaboration and increased citations

- maximum visibility for your research: over $100 \mathrm{M}$ website views per year

At BMC, research is always in progress.

Learn more biomedcentral.com/submissions 